\title{
Article
}

\section{Synthesis, spectral evaluation and pharmacological screening of some sulfa drugs}

\author{
Aziz-Ur-Rehman ${ }^{1, *}$, Muhammad Athar Abbasi ${ }^{1}$, Sabahat Zahra Siddiqui ${ }^{1}$, Shahid Rasool ${ }^{1}$, Sadia Jabeen ${ }^{1}$, \\ Amar Masood Joyia ${ }^{1}$ and Asma Salah-Ud-Din Gondal ${ }^{1}$ \\ 1 Department of Chemistry, Government College University, Lahore-54000, Pakistan.; azizryk@yahoo.com(A.R); \\ atrabbasi@yahoo.com(M.A.A); sabahat.gcu@gmail.com(S.Z.S); shahid786.lhr@gmail.com(S.R); \\ misssadiajabeen@gmail.com(S.J); joyaamir92@gmail.com(A.M.J); asmagonda199@gmail.com(A.S.D.G) \\ * Correspondence: azizryk@yahoo.com
}

Received: 25 January 2019; Accepted: 9 March 2019; Published: 30 June 2019.

\begin{abstract}
Sulfonamides are considered to be biologically active and an important class of pharmaceutical compounds. These constitute an important class of antimicrobials, antidiabetics, anticancer and diuretics. These were the first chemotherapeutic drugs introduced to the world but their rapidly developed resistance decreased their use. In the present work, N-(2/4-substitutedphenyl)-4-(substituted)benzenesulfonamide (3a-d) and $\mathrm{N}$-(tetrahydrofuran-2-ylmethyl)-4-methylbenzenesulfonamide (3e) were synthesized by the reaction of substituted aniline (1a-c) and tetrahydrofuran-2-ylmethanamine (1d) with substituted benzenesulfonyl chloride (2a-d) using $10 \%$ aqueous $\mathrm{Na}_{2} \mathrm{CO}_{3}$ solution as a reaction medium. In the second step, the synthesized molecules 3a-e were allowed to react different alkyl/aralkyl halides (4a-j) to synthesize the target $\mathrm{N}$-substituted compounds, $\mathbf{5 a - z}, \mathbf{a a}, \mathbf{b b}$, using lithium hydride as an activator and $\mathrm{N}, \mathrm{N}$-dimethylformamide (DMF) as a reaction medium. All the synthesized compounds were characterized by using spectral techniques such as ${ }^{1} \mathrm{H}-\mathrm{NMR}$, IR and EI-MS; and further examined for their anti-bacterial activities.
\end{abstract}

Keywords: Antibacterial activity; substituted anilines; sulfonamides.

\section{Introduction}

S ulfonamides $\left(\mathrm{SO}_{2}-\mathrm{NH}-\right)$ have great importance in medicinal chemistry for the synthesis of pharmaceutical compounds with a broad spectrum of biological activities [1]. Sulfonamide drugs with various biological activities have wide applications in the field of medicine for antibacterial agents [2], hypoglycemic [3], diuretics [4], anti-inflammatory [5], hypersensitivity or allergic reactions [6], anticancer agent [7], antifungal [8] as well as antiviral activities [9]. Overdose of sulfonamide with supplementary drug lead brutal occurrence or even fatal intoxication [10]. The work being proceeded by our group [11], the presented work was to introduce new potent molecules against three gram-negative and two gram-positive bacteria. The attempt concluded that the derived molecules executed moderate inhibitory potential.

\section{Experimental}

\subsection{General}

Substituted anilines, substituted benzenesulfonyl chloride and all alkyl/aralkyl halides were purchased from Alfa Aesar, Sigma Aldrick and Merck through local suppliers and were processed without further purification. The solvents used were of analytical grade. Progress of the reactions were monitored by pre-coated TLC silica gel $G-25-U V_{254}$ plates using n-hexane and ethyl acetate in $70: 30$ ratio as solvent system. Gallonkamp melting point apparatus was used to check out melting points by open capillary tube and were uncorrected. Bruker spectrometer was used to record ${ }^{1} \mathrm{H}-\mathrm{NMR}$ spectra in $\mathrm{HCl}_{3}-d 1$ operating at 400 $\mathrm{MHz}$ at $25 \mathrm{oC}$. The chemical shifts are given in ppm (parts per million) and coupling constant in hertz (Hz). MIDAC M 2000 spectrometer was used to record IR spectra. Finnigan MAT-312 instrument along with data system was used to measure Mass spectra (EIMS). 


\subsection{Procedure for the synthesis of N-(2/4-substitutedphenyl)-4-(substituted)benzenesulfonamide (3a-d)} and N-(tetrahydrofuran-2-ylmethyl)-4-methylbenzenesulfonamide (3e)

Substituted aniline (1a-c) and tetrahydrofuran-2-ylmethanamine (1d) (1.1 mmol) were taken in a $100 \mathrm{~mL}$ round bottom flask followed by the addition of $10-15 \mathrm{~mL}$ of distilled water. Mixture was kept on stirring. Then substituted benzenesulfonyl chlorides $(\mathbf{2 a}-\mathbf{d}, 1.1 \mathrm{mmol})$ were added on continuous stirring and $\mathrm{pH}$ was maintained from $9-10$ by adding aq. $\mathrm{Na}_{2} \mathrm{CO}_{3}$. The system is left for shaking for about $3-4$ hours. The purity as well as progress of the reaction was checked out by thin layer chromatography (TLC). After the assurance of completion of reaction by identifying spots, excess distilled water was added to flask. Then the $\mathrm{pH}$ was made acidic (up to a $\mathrm{pH}$ of $4-6$ ) by adding dil. $\mathrm{HCl}$. The greyish white precipitates of title compound were collected by filtration, washed with distilled water and dried for further analysis.

\subsection{General procedure for the synthesis of $\mathrm{N}$-alkyl/aralkyl substituted sulfonamides $(5 \mathrm{a}-\mathrm{z}, \mathrm{aa}, \mathrm{bb})$}

The calculated amount of 3a-e $(0.6 \mathrm{mmol})$ was taken in a $50 \mathrm{~mL}$ round bottom flask and $10 \mathrm{~mL}$ of $\mathrm{DMF}$ is added to dissolve it. Lithium hydride $(0.004 \mathrm{~g}, \mathrm{LiH})$ was also added as an activator to activate 3a-e for further reaction. The mixture was kept on stirring for about $30-45$ minutes at $25^{\circ} \mathrm{C}$ followed by the addition of alkyl/aralkyl halides $(\mathbf{4 a - j}, 0.6 \mathrm{mmol})$. The reaction mixture was left for stirring for about $4-5$ hours. Completion of reaction was confirmed by frequently performed TLC till appearance of single spot. After confirmation, ice cold water was added followed by the addition of 2 drops of $\mathrm{NaOH}$ to make the $\mathrm{pH}$ of reaction mixture basic. Depending upon the nature of product, final products were collected by filtration or solvent extraction (using $\mathrm{CHCl}_{3}$ ).

\subsubsection{4-Acetamido-N-(2-propyl)-N-(2-ethylphenyl)benzenesulfonamide (3a)}

Grayish white amorphous solid; Yield: 75\%; M.P: $185-186^{\circ}$ C; M.F.: $\mathrm{C}_{16} \mathrm{H}_{18} \mathrm{~N}_{2} \mathrm{O}_{3}$ S; M.W.: 318 g/mol; IR $\left(\mathrm{KBr}, v_{\max }\left(\mathrm{cm}^{-1}\right)\right): 3431,3016,1666,1586,1411 ;{ }^{1} \mathrm{H}-\mathrm{NMR}\left(400 \mathrm{MHz}, C D \mathrm{Cl}_{3}, \delta(\mathrm{ppm})\right): 7.66(\mathrm{~d}, \mathrm{~J}=8.8 \mathrm{~Hz}, 2 \mathrm{H}$, H-3', $\left.\mathrm{H}-5^{\prime}\right), 7.56$ (d , J = 8.8 Hz, 2H, H-2' , H-6'), 7.11-7.10 (m, 4H, H-3 to H-6), 2.31 (q, J = 7.6 Hz, 2H, CH. -7 ), $2.18\left(\mathrm{~s}, 3 \mathrm{H}, \mathrm{CH}_{3}-8^{\prime}\right), 1.04\left(\mathrm{t}, \mathrm{J}=7.6 \mathrm{~Hz}, 3 \mathrm{H}, \mathrm{CH}_{3}-8\right)$; EI-MS (m/z): 318, 119, 134, 198.

\subsubsection{N-(4-Ethylphenyl)-4-chlorobenzenesulfonamide (3b)}

Yellowish brown amorphous solid; Yield: 72\%; M.P.: $123-124^{\circ}$ C; M.F.: $C_{14} H_{14} C l N O_{2}$ S; M.W.: 295 $\mathrm{gmol}^{-1}$; IR (KBr, $\left.v_{\max }\left(\mathrm{cm}^{-1}\right)\right): 3255,2938,1590,1440,1030,685 ;{ }^{1} \mathrm{H}-\mathrm{NMR}\left(400 \mathrm{MHz}, \mathrm{CDCl}_{3}, \delta(\mathrm{ppm})\right): 7.59$ (d, $\left.\mathrm{J}=8.8 \mathrm{~Hz}, 2 \mathrm{H}, \mathrm{H}-2^{\prime}, \mathrm{H}-6^{\prime}\right), 7.38\left(\mathrm{~d}, \mathrm{~J}=8.8 \mathrm{~Hz}, 2 \mathrm{H}, \mathrm{H}-3^{\prime}, \mathrm{H}-5^{\prime}\right), 7.06(\mathrm{~d}, \mathrm{~J}=8.4 \mathrm{~Hz}, 2 \mathrm{H}, \mathrm{H}-2, \mathrm{H}-6), 6.94(\mathrm{~d}, \mathrm{~J}=8.4$ Hz, 2H, H-3, H-5), 2.57 (q, J = 7.6 Hz, 2H, H-7), 1.71 (t, J = 7.6 Hz, 3H, H-8); EI-MS (m/z): 297, 295, 175, 119, $111,105,85,76,51$.

\subsubsection{N-(4-Ethoxyphenyl)-4-chlorobenzenesulfonamide (3c)}

Light grey amorphous solid; Yield: 79\%; M.P.: $128-129^{\circ} \mathrm{C}$; M.F.: $\mathrm{C}_{14} \mathrm{H}_{14} \mathrm{ClNO}_{3}$ S; M.W.: $311 \mathrm{gmol}$; IR $\left(\mathrm{KBr}, v_{\max }\left(\mathrm{cm}^{-1}\right)\right): 3245,2916,1587,1415,1133,1061,685 ;{ }^{1} \mathrm{H}-\mathrm{NMR}\left(400 \mathrm{MHz}, \mathrm{CDCl}_{3}, \delta(\mathrm{ppm})\right): 7.59(\mathrm{~d}$, $\left.\mathrm{J}=8.4 \mathrm{~Hz}, 2 \mathrm{H}, \mathrm{H}-2^{\prime}, \mathrm{H}-6^{\prime}\right), 7.37\left(\mathrm{~d}, \mathrm{~J}=8.4 \mathrm{~Hz}, 2 \mathrm{H}, \mathrm{H}-3^{\prime}, \mathrm{H}-5^{\prime}\right), 6.92(\mathrm{~d}, \mathrm{~J}=8.8 \mathrm{~Hz}, 2 \mathrm{H}, \mathrm{H}-2, \mathrm{H}-6), 6.74$ $(\mathrm{d}, \mathrm{J}=8.8 \mathrm{~Hz}, 2 \mathrm{H}, \mathrm{H}-3, \mathrm{H}-5), 3.95$ (q, J = $7.2 \mathrm{~Hz}, 2 \mathrm{H}, \mathrm{H}-7), 1.37$ (t, J = $7.2 \mathrm{~Hz}, 3 \mathrm{H}, \mathrm{H}-8)$; EI-MS (m/z): $313,311,175,136,121,111,92,85,76,51$.

\subsubsection{N-(4-Ethoxyphenyl)benzenesulfonamide (3d)}

Grayish dusty brown amorphous solid; Yield: $78 \%$; M.P.: $128-130^{\circ}$ C; M.F.: $C_{14} H_{15} N O_{3} S$; M.W.: $277 \mathrm{gmol}^{-1}$; IR (KBr, $\left.v_{\max }\left(\mathrm{cm}^{-1}\right)\right): 3266,2982,1594,1445,1153 ;{ }^{1} \mathrm{H}-\mathrm{NMR}\left(400 \mathrm{MHz}, \mathrm{CDCl}_{3}, \delta(\mathrm{ppm})\right): 7.67$ $\left(\mathrm{d}, \mathrm{J}=7.6 \mathrm{~Hz}, 2 \mathrm{H}, \mathrm{H}-2^{\prime} \& \mathrm{H}-6^{\prime}\right), 7.51\left(\mathrm{t}, \mathrm{J}=7.6 \mathrm{~Hz},{ }^{1} \mathrm{H}, \mathrm{H}-4^{\prime}\right), 7.41\left(\mathrm{t}, \mathrm{J}=7.2 \mathrm{~Hz}, 2 \mathrm{H}, \mathrm{H}-3^{\prime} \& \mathrm{H}-5^{\prime}\right), 6.92(\mathrm{~d}, \mathrm{~J}=$ $8.8 \mathrm{~Hz}, 2 \mathrm{H}, \mathrm{H}-2$ \& H-6), 6.73 (d, J = 8.8 Hz, 2H, H-3 \& H-5), 3.94 (q, J = 7.2 Hz, 2H, H-7), 1.36 (t, J = 6.8 Hz, 3H, $\left.\mathrm{CH}_{3}-8\right)$; $\operatorname{EIMS}(\mathrm{m} / \mathrm{z}): 277,141,136,121,91,77,76,51$

\subsubsection{N-(Tetrahydrofuran-2-ylmethyl)-4-methylbenzenesulfonamide (3e)}

White amorphous powder; Yield: 82\%; M.P: $210-211^{\circ} \mathrm{C}$; M.F.: $\mathrm{C}_{12} \mathrm{H}_{17} \mathrm{NO}_{3} \mathrm{~S}$; M.W.: $255 \mathrm{gmol}^{-1}$; IR (KBr, $\left.v_{\max }\left(\mathrm{cm}^{-1}\right)\right): 3463,3046,1620,1383,1187 ;{ }^{1} \mathrm{H}-\mathrm{NMR}\left(400 \mathrm{MHz}, \mathrm{CDCl}_{3}, \delta(\mathrm{ppm})\right): 7.72\left(\mathrm{~d}, \mathrm{~J}=8.4 \mathrm{~Hz}, 2 \mathrm{H}, \mathrm{H}-2^{\prime} \&\right.$ 
H-6' $), 7.29\left(\mathrm{~d}, \mathrm{~J}=8.0 \mathrm{~Hz}, 2 \mathrm{H}, \mathrm{H}-3^{\prime} \& \mathrm{H}-5^{\prime}\right), 3.93-3.87\left(\mathrm{~m}, 1 \mathrm{H}, H_{e}-5\right), 3.78\left(\mathrm{dd}, \mathrm{J}=6.8,8.0 \mathrm{~Hz}, 1 \mathrm{H}, H_{a}-6\right), 3.68(\mathrm{dd}$, $\left.\mathrm{J}=6.8,8.0 \mathrm{~Hz}, 1 \mathrm{H}, H_{b}-6\right), 3.10-3.07(\mathrm{~m}, 1 \mathrm{H}, \mathrm{H}-2), 2.88-2.84\left(\mathrm{~m}, 1 \mathrm{H}, \mathrm{H}_{a}-5\right), 2.40\left(\mathrm{~s}, 3 \mathrm{H}, \mathrm{CH}_{3}-7^{\prime}\right), 1.93-1.80(\mathrm{~m}, 3 \mathrm{H}$, $H_{e}-3$ \& H-4), 1.63-1.53 (m, 1H, $\left.H_{a}-3\right)$; EIMS (m/z): 255, 254, 190, 155, 100, 91, 85, 76, 71, 50.

2.3.6. 4-Acetamido-N-(2-propyl)-N-(2-ethylphenyl)benzenesulfonamide (5a)

Light brown amorphous solid; Yield: $83 \%$; M.P: $113-114^{\circ}$ C; M.F.: $\mathrm{C}_{19} \mathrm{H}_{24} \mathrm{~N}_{2} \mathrm{O}_{3}$ S; M.W.: 360 g/mol; IR $\left(\mathrm{KBr}, v_{\max }\left(\mathrm{cm}^{-1}\right)\right): 3428,3018,1668,1590,1408 ;{ }^{1} \mathrm{H}-\mathrm{NMR}\left(400 \mathrm{MHz}, \mathrm{CDCl}_{3}, \delta(\mathrm{ppm})\right): 7.64(\mathrm{~d}, \mathrm{~J}=8.4 \mathrm{~Hz}, 2 \mathrm{H}$, H-3', H-5'), 7.53 (d, J = 8.4 Hz, 2H, H-2' ${ }^{\prime}$ H-6'), 7.14-7.11 (m, 4H, H-3 to H-6), 4.51 (sep, J = 7.2 Hz, 1H, H-1"'), $2.31\left(\mathrm{q}, \mathrm{J}=7.2 \mathrm{~Hz}, 2 \mathrm{H}, \mathrm{CH}_{2}-7\right), 2.19\left(\mathrm{~s}, 3 \mathrm{H}, \mathrm{CH}_{3}-8^{\prime}\right), 1.07\left(\mathrm{~d}, \mathrm{~J}=6.8 \mathrm{~Hz}, 6 \mathrm{H}, \mathrm{CH}_{3}-2^{\prime \prime}, \mathrm{CH}_{3}-3^{\prime \prime}\right), 1.03(\mathrm{t}, \mathrm{J}=$ $\left.7.2 \mathrm{~Hz}, 3 \mathrm{H}, \mathrm{CH}_{3}-8\right), 0.93\left(\mathrm{~d}, \mathrm{~J}=6.8 \mathrm{~Hz}, 3 \mathrm{H}, \mathrm{CH}_{3}-3^{\prime \prime}\right)$; EI-MS (m/z): 360, 119, 134, 198, 43.

\subsubsection{4-Acetamido-N-(2-butyl)-N-(2-ethylphenyl)benzenesulfonamide (5b)}

Light brown amorphous solid; Yield: $81 \%$; M.P: $134-135^{\circ}$ C; M.F.: $\mathrm{C}_{20} \mathrm{H}_{26} \mathrm{~N}_{2} \mathrm{O}_{3}$ S; M.W.: 374 g/mol; IR $\left(\mathrm{KBr}, v_{\max }\left(\mathrm{cm}^{-1}\right)\right): 3435,3020,1660,1587,1415 ;{ }^{1} \mathrm{H}-\mathrm{NMR}\left(400 \mathrm{MHz}, \mathrm{CDCl}_{3}, \delta(\mathrm{ppm})\right): 7.61(\mathrm{~d}, \mathrm{~J}=8.8 \mathrm{~Hz}, 2 \mathrm{H}$, H-3' $\left.{ }^{\prime}, \mathrm{H}-5^{\prime}\right), 7.56\left(\mathrm{~d}, \mathrm{~J}=8.8 \mathrm{~Hz}, 2 \mathrm{H}, \mathrm{H}-2^{\prime}, \mathrm{H}^{\prime} 6^{\prime}\right), 7.13-7.11$ (m, 4H, H-3 to H-6), 4.23 (sex, J = 6.8 Hz, 1H, H-1"'), $2.36\left(\mathrm{q}, \mathrm{J}=7.2 \mathrm{~Hz}, 2 \mathrm{H}, \mathrm{CH}_{2}-7\right), 2.16\left(\mathrm{~s}, 3 \mathrm{H}, \mathrm{CH}_{3}-8^{\prime}\right), 1.02\left(\mathrm{t}, \mathrm{J}=7.2 \mathrm{~Hz}, 3 \mathrm{H}, \mathrm{CH}_{3}-8\right), 1.00(\mathrm{~d}, \mathrm{~J}=6.8 \mathrm{~Hz}, 3 \mathrm{H}$, $\left.\mathrm{CH}_{3}-4^{\prime \prime}\right), 0.92-0.86\left(\mathrm{~m}, 2 \mathrm{H}, \mathrm{H}-2^{\prime \prime}\right), 0.75\left(\mathrm{t}, \mathrm{J}=6.8 \mathrm{~Hz}, 3 \mathrm{H}, \mathrm{CH}_{3}-3^{\prime \prime}\right)$; EI-MS (m/z): 374, 119, 134, 198, 57.

2.3.8. 4-Acetamido-N-(1-pentyl)-N-(2-ethylphenyl)benzenesulfonamide (5c)

Dark brown amorphous solid; Yield: $77 \%$; M.P: $158-159^{\circ}$ C; M.F.: $\mathrm{C}_{21} \mathrm{H}_{28} \mathrm{~N}_{2} \mathrm{O}_{3}$ S; M.W.: $388 \mathrm{~g} / \mathrm{mol}$; IR $\left(\mathrm{KBr}, v_{\max }\left(\mathrm{cm}^{-1}\right)\right): 3433,3019,1662,1585,1410 ;{ }^{1} \mathrm{H}-\mathrm{NMR}\left(400 \mathrm{MHz}, \mathrm{CDCl}_{3}, \delta(\mathrm{ppm})\right): 7.65(\mathrm{~d}, \mathrm{~J}=8.4 \mathrm{~Hz}, 2 \mathrm{H}$, H-3' ${ }^{\prime}$ H-5'), 7.58 (d, J = 8.4 Hz, 2H, H-2' , H-6'), 7.14-7.12 (m, 4H, H-3 to H-6), 3.64 (t, J = 7.2 Hz, 2H, H-1' $), 2.37$ $\left(\mathrm{q}, \mathrm{J}=7.6 \mathrm{~Hz}, 2 \mathrm{H}, \mathrm{CH}_{2}-7\right), 2.17\left(\mathrm{~s}, 3 \mathrm{H}, \mathrm{CH}_{3}-8^{\prime}\right), 1.36-1.18\left(\mathrm{~m}, 6 \mathrm{H}, \mathrm{H}-2^{\prime \prime}\right.$ to $\left.\mathrm{H}-4^{\prime \prime}\right), 1.04\left(\mathrm{t}, \mathrm{J}=7.6 \mathrm{~Hz}, 3 \mathrm{H}, \mathrm{CH}_{3}-8\right)$, $0.82\left(\mathrm{t}, \mathrm{J}=7.2 \mathrm{~Hz}, 3 \mathrm{H}, \mathrm{CH}_{3}-5^{\prime \prime}\right)$; EI-MS (m/z): 388, 119, 134, 198, 71.

\subsubsection{4-Acetamido-N-benzyl-N-(2-ethylphenyl)benzenesulfonamide (5d)}

Brownish white amorphous solid; Yield: 79\%; M.P: $125-126^{\circ}$ C; M.F.: $\mathrm{C}_{23} \mathrm{H}_{24} \mathrm{~N}_{2} \mathrm{O}_{3}$ S; M.W.: 408 g/mol; IR $\left(\mathrm{KBr}, v_{\max }\left(\mathrm{cm}^{-1}\right)\right): 3430,3015,1664,1584,1412 ;{ }^{1} \mathrm{H}-\mathrm{NMR}\left(400 \mathrm{MHz}, \mathrm{CDCl}_{3}, \delta(\mathrm{ppm})\right): 7.18(\mathrm{~d}, \mathrm{~J}=8.0 \mathrm{~Hz}$, 2H, H-3' $\left.{ }^{\prime} \mathrm{H}-5^{\prime}\right), 7.17\left(\mathrm{~d}, \mathrm{~J}=8.0 \mathrm{~Hz}, 2 \mathrm{H}, \mathrm{H}-2^{\prime}, \mathrm{H}^{\prime} 6^{\prime}\right), 1$ 7.10-7.08 (m, 5H, H-2" to H-6 $\left.{ }^{\prime \prime}\right), 7.02-7.00(\mathrm{~m}, 4 \mathrm{H}, \mathrm{H}-3$ to $\mathrm{H}-6), 4.89\left(\mathrm{~d}, \mathrm{~J}=13.2 \mathrm{~Hz}, 1 \mathrm{H}, H_{\alpha}-7^{\prime \prime}\right), 4.20\left(\mathrm{~d}, \mathrm{~J}=13.2 \mathrm{~Hz}, 1 \mathrm{H}, H_{\beta}-7^{\prime \prime}\right)$, 2.56-2.50 (m, 1H, $\left.H_{\alpha}-7\right)$, 2.35-2.30 (m, $\left.1 \mathrm{H}, \mathrm{H}_{\beta}-7\right), 2.20\left(\mathrm{~s}, 3 \mathrm{H}, \mathrm{CH}_{3}-8^{\prime}\right), 0.87\left(\mathrm{t}, \mathrm{J}=7.2 \mathrm{~Hz}, 3 \mathrm{H}, \mathrm{CH}_{3}-8\right)$, EI-MS (m/z): 318, 119, 134, 198 , 91, 65, 77, 51.

2.3.10. 4-Acetamido-N-(2-chlorobenzyl)-N-(2-ethylphenyl)benzenesulfonamide (5e)

Dark brown amorphous solid; Yield: 84\%; M.P: $130-131^{\circ}$ C; M.F.: $\mathrm{C}_{23} \mathrm{H}_{23} \mathrm{ClN}_{2} \mathrm{O}_{3}$ S; M.W.: 442 g/mol; IR $\left(\mathrm{KBr}, v_{\max }\left(\mathrm{cm}^{-1}\right)\right): 3429,3014,1665,1584,1409,665 ;{ }^{1} \mathrm{H}-\mathrm{NMR}\left(400 \mathrm{MHz}, \mathrm{CDCl}_{3}, \delta(\mathrm{ppm})\right): 7.46(\mathrm{~d}, \mathrm{~J}=8.0 \mathrm{~Hz}$, $\left.2 \mathrm{H}, \mathrm{H}-3^{\prime}, \mathrm{H}-5^{\prime}\right), 7.38\left(\mathrm{~d}, \mathrm{~J}=8.0 \mathrm{~Hz}, 2 \mathrm{H}, \mathrm{H}-2^{\prime}, \mathrm{H}-6^{\prime}\right), 7.34\left(\mathrm{dd}, \mathrm{J}=8.8,3.6 \mathrm{~Hz}, 1 \mathrm{H}, \mathrm{H}-3^{\prime \prime}\right), 7.17(\mathrm{dd}, \mathrm{J}=8.4,4.0 \mathrm{~Hz}$, 1H, H-6" $\left.{ }^{\prime \prime}\right)$ 7.10-7.08 (m, 2H, H-4" $\left.{ }^{\prime \prime} \mathrm{H}-5^{\prime \prime}\right), 7.01-6.99\left(\mathrm{~m}, 4 \mathrm{H}, \mathrm{H}-3\right.$ to H-6), $4.93\left(\mathrm{~d}, \mathrm{~J}=14.0 \mathrm{~Hz}, 1 \mathrm{H}, \mathrm{H}_{\alpha}-7^{\prime \prime}\right), 4.20$ $\left(\mathrm{d}, \mathrm{J}=14.0 \mathrm{~Hz}, 1 \mathrm{H}, H_{\beta}-7^{\prime \prime}\right), 2.53-2.51\left(\mathrm{~m}, 1 \mathrm{H}, H_{\alpha}-7\right), 2.39-2.34\left(\mathrm{~m}, 1 \mathrm{H}, \mathrm{H}_{\beta}-7\right), 2.21\left(\mathrm{~s}, 3 \mathrm{H}, \mathrm{CH}_{3}-8^{\prime}\right), 0.85(\mathrm{t}, \mathrm{J}=$ 7.6 Hz, 3H, $\left.\mathrm{CH}_{3}-8\right)$; EI-MS (m/z): 444, 442, 119, 134, 198, 125, 111.

\subsubsection{4-Acetamido-N-(4-chlorobenzyl)-N-(2-ethylphenyl)benzenesulfonamide (5f)}

Pinkish white amorphous solid; Yield: $76 \%$; M.P: $176-177^{\circ}$ C; M.F.: $\mathrm{C}_{23} \mathrm{H}_{23} \mathrm{ClN}_{2} \mathrm{O}_{3}$ S; M.W.: $442 \mathrm{~g} / \mathrm{mol}$; IR $\left(\mathrm{KBr}, v_{\max }\left(\mathrm{cm}^{-1}\right)\right): 3432,3017,1669,1589,1408,670 ;{ }^{1} \mathrm{H}-\mathrm{NMR}\left(400 \mathrm{MHz}, \mathrm{CDCl}_{3}, \delta(\mathrm{ppm})\right): 7.19(\mathrm{~d}, \mathrm{~J}=8.4 \mathrm{~Hz}$,

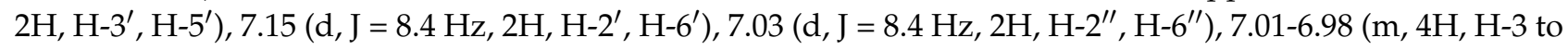
$\mathrm{H}-6), 6.57\left(\mathrm{~d}, \mathrm{~J}=8.0 \mathrm{~Hz}, 2 \mathrm{H}, \mathrm{H}-3^{\prime \prime}, \mathrm{H}-6^{\prime \prime}\right), 4.91\left(\mathrm{~d}, \mathrm{~J}=13.6 \mathrm{~Hz}, 1 \mathrm{H}, \mathrm{H}_{\alpha}-7^{\prime \prime}\right), 4.18\left(\mathrm{~d}, \mathrm{~J}=13.6 \mathrm{~Hz}, 1 \mathrm{H}, H_{\beta}-7^{\prime \prime}\right)$, 2.55-2.49 (m, 1H, $\left.\mathrm{H}_{\alpha}-7\right), 2.37-2.32\left(\mathrm{~m}, 1 \mathrm{H}, \mathrm{H}_{\beta}-7\right), 2.22\left(\mathrm{~s}, 3 \mathrm{H}, \mathrm{CH}_{3}-8^{\prime}\right), 0.86\left(\mathrm{t}, \mathrm{J}=7.6 \mathrm{~Hz}, 3 \mathrm{H}, \mathrm{CH}_{3}-8\right)$; EI-MS (m/z): 444, 442, 119, 134, 198, 90, 76, 99. 


\subsubsection{N-(2-Chloroethyl)-N-(4-ethylphenyl)-4-chlorobenzenesulfonamide (5g)}

Brown amorphous solid; Yield: 77\%; M.P.: $107-108^{\circ}$ C; M.F.: $\mathrm{C}_{16} \mathrm{H}_{17} \mathrm{Cl}_{2} \mathrm{NO}_{2}$ S; M.W.: $357 \mathrm{gmol}^{-1}$; IR $\left(\mathrm{KBr}, v_{\max }\left(\mathrm{cm}^{-1}\right)\right): 2940,1597,1450,1065,693 ;{ }^{1} \mathrm{H}-\mathrm{NMR}\left(400 \mathrm{MHz}, C D C l_{3}, \delta(\mathrm{ppm})\right): 7.52(\mathrm{~d}, \mathrm{~J}=8.4 \mathrm{~Hz}, 2 \mathrm{H}$, H-2' ${ }^{\prime}$ H-6' $), 7.36$ (d, J = 8.0 Hz, 2H, H-3', H-5'), 7.08 (d, J = 8.8 Hz, 2H, H-2, H-6), 6.96 (d, J = 8.0 Hz, 2H, H-3, $\mathrm{H}-5), 3.83\left(\mathrm{t}, \mathrm{J}=7.6 \mathrm{~Hz}, 2 \mathrm{H}, \mathrm{H}-1^{\prime \prime}\right), 3.32\left(\mathrm{t}, \mathrm{J}=6.8 \mathrm{~Hz}, 2 \mathrm{H}, \mathrm{H}-2^{\prime \prime}\right), 2.59(\mathrm{q}, \mathrm{J}=7.2 \mathrm{~Hz}, 2 \mathrm{H}, \mathrm{H}-7), 1.75(\mathrm{t}, \mathrm{J}=7.2 \mathrm{~Hz}$, 3H, H-8); EI-MS (m/z): 361, 359, 357, 175, 119, 111, 105, 85, 76, 63, 51.

\subsubsection{N-(2-Bromoethyl)-N-(4-ethylphenyl)-4-chlorobenzenesulfonamide (5h)}

Light brown amorphous solid; Yield: $82 \%$; M.P.: $81-82^{\circ}$ C; M.F.: $\mathrm{C}_{16} \mathrm{H}_{7} \mathrm{BrClNO}_{2}$ S; M.W.: $401 \mathrm{gmol}^{-1}$; IR $\left(\mathrm{KBr}, v_{\max }\left(\mathrm{cm}^{-1}\right)\right):$ 2950, 1580, 1430, 1070, 700, 587; ${ }^{1} \mathrm{H}-\mathrm{NMR}\left(400 \mathrm{MHz}, \mathrm{CDCl}_{3}, \delta(\mathrm{ppm})\right): 7.53(\mathrm{~d}, \mathrm{~J}=8.4 \mathrm{~Hz}$, $\left.2 \mathrm{H}, \mathrm{H}-2^{\prime}, \mathrm{H}-6^{\prime}\right), 7.36\left(\mathrm{~d}, \mathrm{~J}=8.0 \mathrm{~Hz}, 2 \mathrm{H}, \mathrm{H}-3^{\prime}, \mathrm{H}-5^{\prime}\right), 7.08(\mathrm{~d}, \mathrm{~J}=8.8 \mathrm{~Hz}, 2 \mathrm{H}, \mathrm{H}-2, \mathrm{H}-6), 6.91$ (d, J = 8.0 Hz, 2H, H-3, H-5), $3.81\left(\mathrm{t}, \mathrm{J}=6.8 \mathrm{~Hz}, 2 \mathrm{H}, \mathrm{H}-1^{\prime \prime}\right), 3.38\left(\mathrm{t}, \mathrm{J}=7.6 \mathrm{~Hz}, 2 \mathrm{H}, \mathrm{H}-2^{\prime \prime}\right), 2.55(\mathrm{q}, \mathrm{J}=7.2 \mathrm{~Hz}, 2 \mathrm{H}, \mathrm{H}-7), 1.69(\mathrm{t}, \mathrm{J}=7.6$ Hz, 3H, H-8); EI-MS (m/z): 405, 403, 401, 175, 119, 111, 107, 105, 85, 76, 51.

\subsubsection{N-(2-Chlorobenzyl)-N-(4-ethylphenyl)-4-chlorobenzenesulfonamide (5i)}

Light yellow amorphous solid; Yield: $81 \%$; M.P.: $114-115^{\circ}$ C; M.F.: $\mathrm{C}_{21} \mathrm{H}_{19} \mathrm{Cl}_{2} \mathrm{NO}_{2}$ S; M.W.: $419 \mathrm{gmol}^{-1}$; IR (KBr, $\left.v_{\max }\left(\mathrm{cm}^{-1}\right)\right): 2910,1587,1410,1138,668 ;{ }^{1} \mathrm{H}-\mathrm{NMR}\left(400 \mathrm{MHz}, \mathrm{CDCl}_{3}, \delta(\mathrm{ppm})\right): 7.57(\mathrm{~d}, \mathrm{~J}=8.4 \mathrm{~Hz}, 2 \mathrm{H}$,

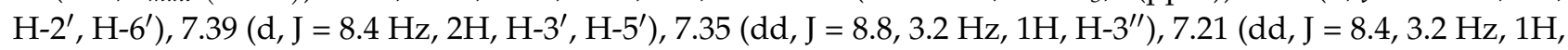
H-6" ${ }^{\prime \prime}, 7.14-7.11\left(\mathrm{~m}, 2 \mathrm{H}, \mathrm{H}-4^{\prime \prime}, \mathrm{H}-5^{\prime \prime}\right), 7.07$ (d, J = 8.4 Hz, 2H, H-2, H-6), $6.92(\mathrm{~d}, \mathrm{~J}=8.4 \mathrm{~Hz}, 2 \mathrm{H}, \mathrm{H}-3, \mathrm{H}-5), 4.47$ (s, 2H, H-7"), 2.58 (q, J = 7.2 Hz, 2H, H-7), 1.73 (t, J = 7.2 Hz, 3H, H-8); EI-MS (m/z): 423, 421, 419, 175, 125, 119, $105,99,90,85,76,51$.

\subsubsection{N-(2-Phenylethyl)-N-(4-ethylphenyl)-4-chlorobenzenesulfonamide (5j)}

Light greyish amorphous solid; Yield: $83 \%$; M.P.: $90-91^{\circ}$ C; M.F.: $\mathrm{C}_{22} \mathrm{H}_{22} \mathrm{ClNO}_{2}$ S; M.W.: $399 \mathrm{gmol}^{-1}$; IR $\left(\mathrm{KBr}, v_{\max }\left(\mathrm{cm}^{-1}\right)\right): 2920,1580,1420,1170,680 ;{ }^{1} \mathrm{H}-\mathrm{NMR}\left(400 \mathrm{MHz}, \mathrm{CDCl}_{3}, \delta(\mathrm{ppm})\right): 7.46(\mathrm{~d}, \mathrm{~J}=8.4 \mathrm{~Hz}, 2 \mathrm{H}$, $\left.\mathrm{H}-2^{\prime}, \mathrm{H}^{\prime} 6^{\prime}\right), 7.36\left(\mathrm{~d}, \mathrm{~J}=8.4 \mathrm{~Hz}, 2 \mathrm{H}, \mathrm{H}-3^{\prime}, \mathrm{H}-5^{\prime}\right), 7.24\left(\mathrm{t}, \mathrm{J}=7.6 \mathrm{~Hz}, 2 \mathrm{H}, \mathrm{H}-3^{\prime \prime}, \mathrm{H}-5^{\prime \prime}\right), 7.18\left(\mathrm{t}, \mathrm{J}=7.6 \mathrm{~Hz}, 1 \mathrm{H}, \mathrm{H}-4^{\prime \prime}\right)$, $7.13\left(\mathrm{~d}, \mathrm{~J}=8.4 \mathrm{~Hz}, 2 \mathrm{H}, \mathrm{H}-2^{\prime \prime}, \mathrm{H}-6^{\prime \prime}\right), 7.08(\mathrm{~d}, \mathrm{~J}=7.2 \mathrm{~Hz}, 2 \mathrm{H}, \mathrm{H}-2, \mathrm{H}-6), 6.92(\mathrm{~d}, \mathrm{~J}=8.0 \mathrm{~Hz}, 2 \mathrm{H}, \mathrm{H}-3, \mathrm{H}-5), 3.73(\mathrm{t}$, $\left.\mathrm{J}=7.2 \mathrm{~Hz}, 2 \mathrm{H}, \mathrm{H}-8^{\prime \prime}\right), 2.75\left(\mathrm{t}, \mathrm{J}=7.2 \mathrm{~Hz}, 2 \mathrm{H}, \mathrm{H}-7^{\prime \prime}\right), 2.64(\mathrm{q}, \mathrm{J}=7.6 \mathrm{~Hz}, 2 \mathrm{H}, \mathrm{H}-7), 1.23(\mathrm{t}, \mathrm{J}=7.6 \mathrm{~Hz}, 3 \mathrm{H}, \mathrm{H}-8)$; EI-MS (m/z): 401, 399, 175, 119, 105, 91, 85, 77, 76, 65, 51.

\subsubsection{N-(3-Phenylpropyl)-N-(4-ethylphenyl)-4-chlorobenzenesulfonamide (5k)}

Yellowish brown amorphous solid; Yield: $80 \%$; M.P.: $85-86^{\circ}$ C; M.F.: $\mathrm{C}_{23} \mathrm{H}_{24} \mathrm{ClNO}_{2}$ S; M.W.: $413 \mathrm{gmol}^{-1}$; IR (KBr, $\left.v_{\max }\left(\mathrm{cm}^{-1}\right)\right): 2975,1590,1415,1155,678 ;{ }^{1} \mathrm{H}-\mathrm{NMR}\left(400 \mathrm{MHz}, \mathrm{CDCl}_{3}, \delta(\mathrm{ppm})\right): 7.56(\mathrm{~d}, \mathrm{~J}=8.4 \mathrm{~Hz}, 2 \mathrm{H}$, $\left.\mathrm{H}-2^{\prime}, \mathrm{H}^{\prime} 6^{\prime}\right), 7.34\left(\mathrm{~d}, \mathrm{~J}=8.4 \mathrm{~Hz}, 2 \mathrm{H}, \mathrm{H}-3^{\prime}, \mathrm{H}-5^{\prime}\right), 7.23\left(\mathrm{t}, \mathrm{J}=7.6 \mathrm{~Hz}, 2 \mathrm{H}, \mathrm{H}-3^{\prime \prime}, \mathrm{H}-5^{\prime \prime}\right), 7.17\left(\mathrm{t}, \mathrm{J}=7.6 \mathrm{~Hz}, 1 \mathrm{H}, \mathrm{H}-4^{\prime \prime}\right)$, $7.09\left(\mathrm{~d}, \mathrm{~J}=7.6 \mathrm{~Hz}, 2 \mathrm{H}, \mathrm{H}-2^{\prime \prime}, \mathrm{H}-6^{\prime \prime}\right), 7.04(\mathrm{~d}, \mathrm{~J}=8.8 \mathrm{~Hz}, 2 \mathrm{H}, \mathrm{H}-2, \mathrm{H}-6), 6.96(\mathrm{~d}, \mathrm{~J}=8.0 \mathrm{~Hz}, 2 \mathrm{H}, \mathrm{H}-3, \mathrm{H}-5), 3.75(\mathrm{t}, \mathrm{J}$ $\left.=6.8 \mathrm{~Hz}, 2 \mathrm{H}, \mathrm{H}-9^{\prime \prime}\right), 2.65\left(\mathrm{t}, \mathrm{J}=7.6 \mathrm{~Hz}, 2 \mathrm{H}, \mathrm{H}-7^{\prime \prime}\right), 2.55(\mathrm{q}, \mathrm{J}=7.2 \mathrm{~Hz}, 2 \mathrm{H}, \mathrm{H}-7), 2.02\left(\right.$ qui, J = 6.8 Hz, 2H, H-8 ${ }^{\prime \prime}$ ), $1.73(\mathrm{t}, \mathrm{J}=7.6 \mathrm{~Hz}, 3 \mathrm{H}, \mathrm{H}-8)$; EI-MS (m/z): 415, 413, 175, 119, 105, 91, 85, 77, 76, 65, 51.

\subsubsection{N-(2-Chloroethyl)-N-(4-ethoxyphenyl)-4-chlorobenzenesulfonamide (5l)}

Light grey amorphous solid; Yield: 79\%; M.P.: 120 - $121^{\circ}$ C; M.F.: $\mathrm{C}_{16} \mathrm{H}_{17} \mathrm{Cl}_{2} \mathrm{NO}_{3}$ S; M.W.: $373 \mathrm{gmol}^{-1}$; IR $\left(\mathrm{KBr}, v_{\max }\left(\mathrm{cm}^{-1}\right)\right): 2900,1600,1455,1185,1065,695 ;{ }^{1} \mathrm{H}-\mathrm{NMR}\left(400 \mathrm{MHz}, C D C l_{3}, \delta(\mathrm{ppm})\right): 7.59(\mathrm{~d}, \mathrm{~J}=8.4 \mathrm{~Hz}$, 2H, H-2', $\left.\mathrm{H}-6^{\prime}\right), 7.39$ (d, J = 8.0 Hz, 2H, H-3', H-5'), 6.90 (d, J = 8.4 Hz, 2H, H-2, H-6), 6.77 (d, J = 8.0 Hz, 2H, H-3, H-5), 3.95 (q, J = 7.2 Hz, 2H, H-7), 3.81 (t, J = 7.2 Hz, 2H, H-1" $), 3.41\left(\mathrm{t}, \mathrm{J}=7.2 \mathrm{~Hz}, 2 \mathrm{H}, \mathrm{H}-2^{\prime \prime}\right) 1.33(\mathrm{t}, \mathrm{J}=7.6$ Hz, 3H, H-8); EI-MS (m/z): 377 , 375, 373, 175, 136, 121, 111, 92, 85, 76, 63, 51.

\subsubsection{N-(2-Bromoethyl)-N-(4-ethoxyphenyl)-4-chlorobenzenesulfonamide (5m)}

Whitish grey amorphous solid; Yield: $84 \%$; M.P.: $90-91^{\circ}$ C; M.F.: $\mathrm{C}_{16} \mathrm{H}_{17} \mathrm{BrClNO}_{3}$ S ; M.W.: $417 \mathrm{gmol}^{-1}$; $\operatorname{IR}\left(\mathrm{KBr}, v_{\max }\left(\mathrm{cm}^{-1}\right)\right): 2935,1600,1410,1100,1050,710,577 ;{ }^{1} \mathrm{H}-\mathrm{NMR}\left(400 \mathrm{MHz}, C D C l_{3}, \delta(\mathrm{ppm})\right): 7.60(\mathrm{~d}, \mathrm{~J}=$ $\left.8.8 \mathrm{~Hz}, 2 \mathrm{H}, \mathrm{H}-2^{\prime}, \mathrm{H}^{\prime} 6^{\prime}\right), 7.37$ (d, J = 8.8 Hz, 2H, H-3' $\left.{ }^{\prime} \mathrm{H}-5^{\prime}\right), 6.94(\mathrm{~d}, \mathrm{~J}=8.4 \mathrm{~Hz}, 2 \mathrm{H}, \mathrm{H}-2, \mathrm{H}-6), 6.76$ (d, J = 8.4 Hz, 2H, H-3, H-5), 3.97 (q, J = 7.6 Hz, 2H, H-7), $3.83\left(\mathrm{t}, \mathrm{J}=7.6 \mathrm{~Hz}, 2 \mathrm{H}, \mathrm{H}-1^{\prime \prime}\right), 3.37\left(\mathrm{t}, \mathrm{J}=6.8 \mathrm{~Hz}, 2 \mathrm{H}, \mathrm{H}-2^{\prime \prime}\right), 1.39(\mathrm{t}, \mathrm{J}$ $=7.6 \mathrm{~Hz}, 3 \mathrm{H}, \mathrm{H}-8)$; EI-MS (m/z): 421, 419, 417, 175, 136, 121, 111, 107, 92, 85, 76, 51. 
2.3.19. N-(2-Chlorobenzyl)-N-(4-ethoxyphenyl)-4-chlorobenzenesulfonamide (5n)

Grayish black amorphous solid; Yield: 82\%; M.P.: $105-106^{\circ}$ C; M.F.: $\mathrm{C}_{21} \mathrm{H}_{19} \mathrm{Cl}_{2} \mathrm{NO}_{3}$ S; M.W.: $435 \mathrm{gmol}^{-1}$; $\operatorname{IR}\left(\mathrm{KBr}, v_{\max }\left(\mathrm{cm}^{-1}\right)\right): 2890,1550,1400,1150,1150,690 ;{ }^{1} \mathrm{H}-\mathrm{NMR}\left(400 \mathrm{MHz}, \mathrm{CDCl}_{3}, \delta(\mathrm{ppm})\right): 7.57(\mathrm{~d}, \mathrm{~J}=8.0$

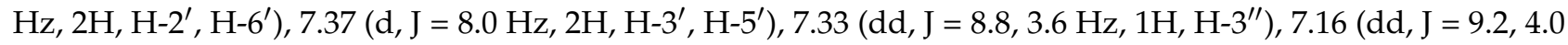
$\left.\mathrm{Hz}, 1 \mathrm{H}, \mathrm{H}-6^{\prime \prime}\right), 7.11-7.09$ (m, 2H, H-4" $\left.{ }^{\prime \prime} \mathrm{H}-5^{\prime \prime}\right), 6.90$ (d, J = 8.4 Hz, 2H, H-2, H-6), 6.72 (d, J = 8.4 Hz, 2H, H-3, H-5), 4.46 (s, 2H, H-7"'), 3.93 (q, J = 6.8 Hz, 2H, H-7), 1.37 (t, J = 6.8 Hz, 3H, H-8); EI-MS (m/z): 449, 437, 435, $175,136,125,121,111,99,92,90,85,76,51$.

\subsubsection{N-(2-Phenylethyl)-N-(4-ethoxyphenyl)-4-chlorobenzenesulfonamide (5o)}

Dark grayish amorphous solid; Yield: $80 \%$; M.P.: $74-75^{\circ}$ C; M.F.: $\mathrm{C}_{22} \mathrm{H}_{22} \mathrm{ClNO}_{3}$ S; M.W.: $415 \mathrm{gmol}^{-1}$; IR $\left(\mathrm{KBr}, v_{\max }\left(\mathrm{cm}^{-1}\right)\right): 2950,1580,1440,1133,1190,700 ;{ }^{1} \mathrm{H}-\mathrm{NMR}\left(400 \mathrm{MHz}, C^{2} C_{3}, \delta(\mathrm{ppm})\right): 7.58(\mathrm{~d}, \mathrm{~J}=8.8 \mathrm{~Hz}$, 2H, H-2' , H-6'), 7.36 (d, J = 8.8 Hz, 2H, H-3', H-5'), 7.19 (t, J = 7.2 Hz, 2H, H-3"', H-5"), 7.16 (t, J = 6.4 Hz, 1H, H-4") 7.05 (d, J = 7.6 Hz, 2H, H-2", H-6"), 6.90 (d, J = 8.4 Hz, 2H, H-2, H-6), 6.73 (d, J = 8.4 Hz, 2H, H-3, H-5), $3.93(\mathrm{q}, \mathrm{J}=7.6 \mathrm{~Hz}, 2 \mathrm{H}, \mathrm{H}-7), 2.85\left(\mathrm{t}, \mathrm{J}=6.8 \mathrm{~Hz}, 2 \mathrm{H}, \mathrm{H}-8^{\prime \prime}\right), 2.61\left(\mathrm{t}, \mathrm{J}=6.8 \mathrm{~Hz}, 2 \mathrm{H}, \mathrm{H}-7^{\prime \prime}\right), 1.34(\mathrm{t}, \mathrm{J}=7.2 \mathrm{~Hz}, 3 \mathrm{H}$, H-8); EI-MS (m/z): 417, 415, 175, 136, 121, 111, 105, 92, 91, 85, 77, 65, 51.

\subsubsection{N-(3-Phenylpropyl)-N-(4-ethoxyphenyl)-4-chlorobenzenesulfonamide (5p)}

Grey amorphous solid; Yield: $81 \%$; M.P.: $80-81^{\circ}$ C; M.F.: $\mathrm{C}_{23} \mathrm{H}_{24} \mathrm{ClNO}_{3} \mathrm{~S}$; M.W.: $429 \mathrm{gmol}^{-1}$; IR (KBr, $\left.v_{\max }\left(\mathrm{cm}^{-1}\right)\right): 3000,1570,1425,1145,1160,670 ;{ }^{1} \mathrm{H}-\mathrm{NMR}\left(400 \mathrm{MHz}, \mathrm{CDCl}_{3}, \delta(\mathrm{ppm})\right): 7.55(\mathrm{~d}, \mathrm{~J}=8.0 \mathrm{~Hz}, 2 \mathrm{H}$, H-2' H-6 $\left.^{\prime}\right), 7.34\left(\mathrm{~d}, \mathrm{~J}=8.0 \mathrm{~Hz}, 2 \mathrm{H}, \mathrm{H}-3^{\prime}, \mathrm{H}^{-5^{\prime}}\right), 7.25\left(\mathrm{t}, \mathrm{J}=7.6 \mathrm{~Hz}, 2 \mathrm{H}, \mathrm{H}-3^{\prime \prime}, \mathrm{H}-5^{\prime \prime}\right), 7.15\left(\mathrm{t}, \mathrm{J}=7.6 \mathrm{~Hz}, 1 \mathrm{H}, \mathrm{H}-4^{\prime \prime}\right)$, $7.03\left(\mathrm{~d}, \mathrm{~J}=7.2 \mathrm{~Hz}, 2 \mathrm{H}, \mathrm{H}-2^{\prime \prime}, \mathrm{H}-6^{\prime \prime}\right), 6.95(\mathrm{~d}, \mathrm{~J}=8.4 \mathrm{~Hz}, 2 \mathrm{H}, \mathrm{H}-2, \mathrm{H}-6), 6.74(\mathrm{~d}, \mathrm{~J}=8.4 \mathrm{~Hz}, 2 \mathrm{H}, \mathrm{H}-3, \mathrm{H}-5), 3.95$ (q, $\mathrm{J}=7.2 \mathrm{~Hz}, 2 \mathrm{H}, \mathrm{H}-7), 3.51\left(\mathrm{t}, \mathrm{J}=6.8 \mathrm{~Hz}, 2 \mathrm{H}, \mathrm{H}-9^{\prime \prime}\right), 2.63\left(\mathrm{t}, \mathrm{J}=8.0 \mathrm{~Hz}, 2 \mathrm{H}, \mathrm{H}-7^{\prime \prime}\right), 1.71$ (qui, J = 7.6, 2H, H-8 $\left.{ }^{\prime \prime}\right), 1.33$ $(\mathrm{t}, \mathrm{J}=6.8 \mathrm{~Hz}, 3 \mathrm{H}, \mathrm{H}-8)$; EI-MS (m/z): 431, 429, 175, 136, 121, 119, 111, 105, 92, 91, 85, 77, 65, 51.

\subsubsection{N-(4-Ethoxyphenyl)-N-propylbenzenesulfonamide (5q)}

Creamy brown amorphous solid; Yield: $83 \%$; M.P.: $70-72^{\circ}$ C; M.F.: $\mathrm{C}_{17} \mathrm{H}_{21} \mathrm{NO}_{3}$ S; M.W.: $319 \mathrm{gmol}^{-1}$; IR $\left(\mathrm{KBr}, v_{\max }\left(\mathrm{cm}^{-1}\right)\right): 2921,1598,1434,1106 ; \mathrm{H}^{1}-\mathrm{NMR}\left(400 \mathrm{MHz}, \mathrm{CDCl}_{3}, \delta(\mathrm{ppm})\right): 7.68\left(\mathrm{~d}, \mathrm{~J}=7.6 \mathrm{~Hz}, 2 \mathrm{H}, \mathrm{H}-2^{\prime}\right.$ \& H-6 $\left.6^{\prime}\right), 7.52\left(\mathrm{t}, \mathrm{J}=7.6 \mathrm{~Hz}, 1 \mathrm{H}, \mathrm{H}-4^{\prime}\right), 7.40\left(\mathrm{t}, \mathrm{J}=7.6 \mathrm{~Hz}, 2 \mathrm{H}, \mathrm{H}-3^{\prime}\right.$ \& H-5'), $6.91(\mathrm{~d}, \mathrm{~J}=8.4 \mathrm{~Hz}, 2 \mathrm{H}, \mathrm{H}-2$ \& H-6), 6.75 (d, J = $8.0 \mathrm{~Hz}, 2 \mathrm{H}, \mathrm{H}-3$ \& H-5), 4.54 (sep, J = 6.8 Hz, 1H, H-1"), 3.91 (q, J = 7.2 Hz, 2H, H-7), 1.37 (t, J = 7.2 Hz, $\left.3 \mathrm{H}, \mathrm{CH}_{3}-8\right), 1.04\left(\mathrm{~d}, \mathrm{~J}=6.8 \mathrm{~Hz}, 6 \mathrm{H}, \mathrm{CH}_{3}-2^{\prime \prime} \& \mathrm{CH}_{3}-3^{\prime \prime}\right)$; EIMS (m/z): 319, 242, 198, 178, 155, 141, 121, 77, 64, 57,45 .

\subsubsection{N-sec-Butyl-N-(4-ethoxyphenyl)benzenesulfonamide (5r)}

Dark brown hygroscopic solid; Yield: $76 \%$; M.P.: $38-40^{\circ}$ C; M.F.: $\mathrm{C}_{18} \mathrm{H}_{23} \mathrm{NO}_{3}$ S; M.W.: $333 \mathrm{gmol}^{-1}$; IR $\left(\mathrm{KBr}, v_{\max }\left(\mathrm{cm}^{-1}\right)\right): 2976,1506,1331,1141 ; \mathrm{H}^{1}-\mathrm{NMR}\left(400 \mathrm{MHz}, \mathrm{CDCl}_{3}, \delta(\mathrm{ppm})\right): 7.66\left(\mathrm{~d}, \mathrm{~J}=8.0 \mathrm{~Hz}, 2 \mathrm{H}, \mathrm{H}-2^{\prime}\right.$ \& H-6' $), 7.53\left(\mathrm{t}, \mathrm{J}=7.6 \mathrm{~Hz}, 1 \mathrm{H}, \mathrm{H}-4^{\prime}\right), 7.41\left(\mathrm{t}, \mathrm{J}=7.6 \mathrm{~Hz}, 2 \mathrm{H}, \mathrm{H}-3^{\prime} \& \mathrm{H}-5^{\prime}\right), 6.92(\mathrm{~d}, \mathrm{~J}=8.0 \mathrm{~Hz}, 2 \mathrm{H}, \mathrm{H}-2$ \& H-6), $6.74\left(\mathrm{~d}, \mathrm{~J}=8.0 \mathrm{~Hz}, 2 \mathrm{H}, \mathrm{H}-3\right.$ \& H-5), $4.25\left(\mathrm{sex}, \mathrm{J}=7.2 \mathrm{~Hz}, 1 \mathrm{H}, \mathrm{H}-1^{\prime \prime}\right), 3.94(\mathrm{q}, \mathrm{J}=7.2 \mathrm{~Hz}, 2 \mathrm{H}, \mathrm{H}-7), 1.35$ (t, J = 6.8 $\left.\mathrm{Hz}, 3 \mathrm{H}, \mathrm{CH}_{3}-8\right), 1.01\left(\mathrm{~d}, \mathrm{ăj} \breve{a}=6.8 \mathrm{~Hz}, 3 \mathrm{H}, \mathrm{CH}_{3}-4^{\prime \prime}\right), 0.90-0.85\left(\mathrm{~m}, 2 \mathrm{H}, \mathrm{H}-2^{\prime \prime}\right), 0.74\left(\mathrm{t}, \mathrm{J}=7.2 \mathrm{~Hz}, 3 \mathrm{H}, \mathrm{CH}_{3}-3^{\prime \prime}\right)$; $\operatorname{EIMS~(m/z):~333,~256,~212,~192,~155,~141,~135,~} 77,71,64,57,45$.

\subsubsection{N-(4-Ethoxyphenyl)-N-pentylbenzenesulfonamide (5s)}

Clay brown amorphous solid; Yield: $83 \%$; M.P.: $90-93^{\circ} \mathrm{C}$; M.F.: $\mathrm{C}_{19} \mathrm{H}_{25} \mathrm{NO}_{3}$ S; M.W.: $347 \mathrm{gmol}^{-1}$; IR (KBr, $\left.v_{\max }\left(\mathrm{cm}^{-1}\right)\right): 2938,1496,1385,1141 ; \mathrm{H}^{1}-\mathrm{NMR}\left(400 \mathrm{MHz}, \mathrm{CDCl}_{3}, \delta(\mathrm{ppm})\right): 7.68\left(\mathrm{~d}, \mathrm{~J}=8.4 \mathrm{~Hz}, 2 \mathrm{H}, \mathrm{H}-2^{\prime} \& \mathrm{H}-6^{\prime}\right)$, $7.54\left(\mathrm{t}, \mathrm{J}=8.0 \mathrm{~Hz}, 1 \mathrm{H}, \mathrm{H}-4^{\prime}\right), 7.42\left(\mathrm{t}, \mathrm{J}=7.6 \mathrm{~Hz}, 2 \mathrm{H}, \mathrm{H}-3^{\prime} \& \mathrm{H}-5^{\prime}\right), 6.91(\mathrm{~d}, \mathrm{~J}=8.4 \mathrm{~Hz}, 2 \mathrm{H}, \mathrm{H}-2$ \& H-6), $6.74(\mathrm{~d}$, $\mathrm{J}=8.4 \mathrm{~Hz}, 2 \mathrm{H}, \mathrm{H}-3$ \& H-5), $3.92(\mathrm{q}, \mathrm{J}=7.2 \mathrm{~Hz}, 2 \mathrm{H}, \mathrm{H}-7), 3.66\left(\mathrm{t}, \mathrm{J}=7.6 \mathrm{~Hz}, 2 \mathrm{H}, \mathrm{H}-1^{\prime \prime}\right), 1.36(\mathrm{t}, \mathrm{J}=7.2 \mathrm{~Hz}, 3 \mathrm{H}$, $\left.\mathrm{CH}_{3}-8\right), 1.34-1.16$ (m, 6H, H-2" to H-4"), 0.80 (t, J = 6.8 Hz, 3H, $\left.\mathrm{CH}_{3}-5^{\prime \prime}\right)$; EIMS (m/z): 347, 263, 239, 206, 199, $162,155,149,141,135,121,85,71,65,64$.

\subsubsection{N-(4-Ethoxyphenyl)-N-benzylbenzenesulfonamide (5t)}

Clay brown amorphous solid; Yield: $92 \%$; M.P.: $104-106^{\circ}$ C; M.F.: $\mathrm{C}_{21} \mathrm{H}_{21} \mathrm{NO}_{3}$ S; M.W.: $367 \mathrm{gmol}^{-1}$; IR $\left(\mathrm{KBr}, v_{\max }\left(\mathrm{cm}^{-1}\right)\right): 2980,1508,1391,1155 ; \mathrm{H}^{1}-\mathrm{NMR}\left(400 \mathrm{MHz}, \mathrm{CDCl}_{3}, \delta(\mathrm{ppm})\right): 7.64\left(\mathrm{~d}, \mathrm{~J}=7.6 \mathrm{~Hz}, 2 \mathrm{H}, \mathrm{H}-2^{\prime}\right.$ \& 
H-6' $), 7.58\left(\mathrm{t}, \mathrm{J}=7.2 \mathrm{~Hz}, 1 \mathrm{H}, \mathrm{H}-4^{\prime}\right), 7.47\left(\mathrm{t}, \mathrm{J}=7.6 \mathrm{~Hz}, 2 \mathrm{H}, \mathrm{H}-3^{\prime} \& \mathrm{H}-5^{\prime}\right), 7.18-7.12\left(\mathrm{~m}, 5 \mathrm{H}, \mathrm{H}-2^{\prime \prime}\right.$ to H-6"), 6.81 (d, $\mathrm{J}=7.6 \mathrm{~Hz}, 2 \mathrm{H}, \mathrm{H}-3$ \& H-5), 6.67 (d, J = 8.4 Hz, 2H, H-2 \& H-6), 4.93 (s, 2H, H-7"), 4.93 (q, J = 7.2 Hz, 2H, H-7), $1.36\left(\mathrm{t}, \mathrm{J}=6.8 \mathrm{~Hz}, 3 \mathrm{H}, \mathrm{CH}_{3}-8\right)$; EIMS (m/z): 367, 155, 141, 135, 121, 105, 91, 77, 76, 51, 45.

\subsubsection{N-(2-Chlorobenzyl)-N-(4-ethoxyphenyl)benzenesulfonamide (5u)}

Shiny crystalline brown solid; Yield: $90 \%$; M.P.: $85-88^{\circ}$ C; M.F.: $\mathrm{C}_{21} \mathrm{H}_{20} \mathrm{ClNO}_{3}$ S; M.W.: $401 \mathrm{gmol}^{-1}$; IR $\left(\mathrm{KBr}, v_{\max }\left(\mathrm{cm}^{-1}\right)\right): 2923,1508,1445,1114690 ; \mathrm{H}^{1}-\mathrm{NMR}\left(400 \mathrm{MHz}, \mathrm{CDCl}_{3}, \delta(\mathrm{ppm})\right): 7.67(\mathrm{~d}, \mathrm{~J}=7.2 \mathrm{~Hz}, 2 \mathrm{H}$, H-2' \& H-6'), 7.51 (t, J = 7.6 Hz, H, H-4' $), 7.41\left(\mathrm{t}, \mathrm{J}=7.2 \mathrm{~Hz}, 2 \mathrm{H}, \mathrm{H}-3^{\prime} \& \mathrm{H}-5^{\prime}\right), 7.36\left(\mathrm{dd}, \mathrm{J}=9.2,3.6 \mathrm{~Hz}, 1 \mathrm{H}, \mathrm{H}-3^{\prime \prime}\right)$, $7.19\left(\mathrm{dd}, \mathrm{J}=9.6,4.0 \mathrm{~Hz}, 1 \mathrm{H}, \mathrm{H}-6^{\prime \prime}\right), 7.12 \mathrm{UU}^{\prime} .10\left(\mathrm{~m}, 2 \mathrm{H}, \mathrm{H}-44^{\prime \prime} \& \mathrm{H}-5^{\prime \prime}\right), 6.92(\mathrm{~d}, \mathrm{~J}=8.8 \mathrm{~Hz}, 2 \mathrm{H}, \mathrm{H}-2$ \& H-6), $6.73(\mathrm{~d}$, $\mathrm{J}=8.8 \mathrm{~Hz}, 2 \mathrm{H}, \mathrm{H}-3$ \& H-5), 4.49 (s, 2H, H-7"), $3.94(\mathrm{q}, \mathrm{J}=7.2 \mathrm{~Hz}, 2 \mathrm{H}, \mathrm{H}-7), 1.36$ (t, J = 6.8 Hz, 3H, CH -8 ); EIMS (m/z): 403, 401, 324, 267, 202, 155, 141, 139, 135, 125, 121, 104, 77, 52.

\subsubsection{N-(4-Chlorobenzyl)-N-(4-ethoxyphenyl)benzenesulfonamide (5v)}

Dusty brown amorphous solid; Yield: $79 \%$; M.P.: $83-85^{\circ} \mathrm{C}$; M.F.: $\mathrm{C}_{21} \mathrm{H}_{20} \mathrm{ClNO}_{3} \mathrm{~S}$; M.W.: $401 \mathrm{gmol}^{-1}$; IR $\left(\mathrm{KBr}, v_{\max }\left(\mathrm{cm}^{-1}\right)\right): 2932,1508,1391,1114,685 ; \mathrm{H}^{1}-\mathrm{NMR}\left(400 \mathrm{MHz}, \mathrm{CDCl}_{3}, \delta(\mathrm{ppm})\right): 7.64(\mathrm{~d}, \mathrm{~J}=7.6 \mathrm{~Hz}, 2 \mathrm{H}$, H-2' \& H-6'), $7.58\left(\mathrm{t}, \mathrm{J}=7.2 \mathrm{~Hz}, 1 \mathrm{H}, \mathrm{H}-4^{\prime}\right), 7.47\left(\mathrm{t}, \mathrm{J}=7.6 \mathrm{~Hz}, 2 \mathrm{H}, \mathrm{H}-3^{\prime} \& \mathrm{H}-5^{\prime}\right), 7.18\left(\mathrm{~d}, \mathrm{~J}=8.4 \mathrm{~Hz}, 2 \mathrm{H}, \mathrm{H}-3^{\prime \prime}\right.$ \& H-5"), $7.13(\mathrm{~d}, \mathrm{~J}=8.0 \mathrm{~Hz}, 2 \mathrm{H}, \mathrm{H}-2 "$ \& H-6"), $6.80(\mathrm{~d}, \mathrm{~J}=6.4 \mathrm{~Hz}, 2 \mathrm{H}, \mathrm{H}-3$ \& H-5), $6.67(\mathrm{~d}, \mathrm{~J}=8.8 \mathrm{~Hz}, 2 \mathrm{H}, \mathrm{H}-2$ \& H-6), 4.63 (s, 2H, H-7"), 4.93 (q, J = 7.2 Hz, 2H, H-7), 1.36 (t, J = 6.8 Hz, 3H, CH3-8); EIMS (m/z): 403, 401, 324, $267,202,155,141,139,135,125,121,104,77,52$.

\subsubsection{N-Pentyl-N-(tetrahydrofuran-2-ylmethyl)-4-methylbenzenesulfonamide (5w)}

Fluorescent green sticky liquid; Yield: $68 \%$; M.F.: $\mathrm{C}_{17} \mathrm{H}_{27} \mathrm{NO}_{3}$ S; M.W.: $325 \mathrm{gmol}^{-1}$; IR $\left(\mathrm{KBr}, v_{\max }\left(\mathrm{cm}^{-1}\right)\right)$ : 3087, 1620, 1383, 1165; ${ }^{1} \mathrm{H}-\mathrm{NMR}\left(400 \mathrm{MHz}, \mathrm{CDCl}_{3}, \delta(\mathrm{ppm})\right): 7.70\left(\mathrm{~d}, \mathrm{~J}=8.4 \mathrm{~Hz}, 2 \mathrm{H}, \mathrm{H}-2^{\prime} \& \mathrm{H}-6^{\prime}\right), 7.32(\mathrm{~d}, \mathrm{~J}=$ $\left.8.4 \mathrm{~Hz}, 2 \mathrm{H}, \mathrm{H}-3^{\prime} \& \mathrm{H}^{-} 5^{\prime}\right), 4.03-3.97\left(\mathrm{~m}, 1 \mathrm{H}, \mathrm{H}_{e}-5\right), 3.82\left(\mathrm{dd}, \mathrm{J}=7.2,8.0 \mathrm{~Hz}, 1 \mathrm{H}, \mathrm{H}_{a}-6\right), 3.72(\mathrm{dd}, \mathrm{J}=6.4,7.6 \mathrm{~Hz}$, $\left.1 \mathrm{H}, \mathrm{H}_{b}-6\right), 3.68\left(\mathrm{t}, \mathrm{J}=8.0 \mathrm{~Hz}, 2 \mathrm{H}, \mathrm{H}-1^{\prime \prime}\right), 3.24-3.17(\mathrm{~m}, 1 \mathrm{H}, \mathrm{H}-2), 3.11-3.07\left(\mathrm{~m}, 1 \mathrm{H}, \mathrm{H}_{a}-5\right), 2.39\left(\mathrm{~s}, 3 \mathrm{H}, \mathrm{CH}_{3}-7^{\prime}\right)$, 1.99-1.96 (m, $\left.1 \mathrm{H}, \mathrm{H}_{e}-3\right), 1.68-1.65\left(\mathrm{~m}, 1 \mathrm{H}, \mathrm{H}_{e}-4\right), 1.56-1.49\left(\mathrm{~m}, 2 \mathrm{H}, \mathrm{H}_{a}-3, \mathrm{H}_{a}-4\right), 1.28-1.15\left(\mathrm{~m}, 6 \mathrm{H}, \mathrm{H}-2^{\prime \prime}\right.$ to $\left.\mathrm{H}-4^{\prime \prime}\right)$, $0.85\left(\mathrm{t}, \mathrm{J}=7.2 \mathrm{~Hz}, 3 \mathrm{H}, \mathrm{H}-5^{\prime \prime}\right)$; EIMS (m/z): 325, 254, 190, 155, 99, 91, 85, 76, 71, 71, 50.

\subsubsection{N-Benzyl-N-(tetrahydrofuran-2-ylmethyl)-4-methylbenzenesulfonamide (5x)}

White sticky solid; Yield: 55\%; M.F.: $\mathrm{C}_{19} \mathrm{H}_{23} \mathrm{NO}_{3} \mathrm{~S}$; M.W.: $345 \mathrm{gmol}^{-1}$; IR $\left(\mathrm{KBr}, v_{\max }\left(\mathrm{cm}^{-1}\right)\right)$ : 2955, 1608, 1386, 1172; ${ }^{1} \mathrm{H}-\mathrm{NMR}\left(400 \mathrm{MHz}, \mathrm{CDCl}_{3}, \delta(\mathrm{ppm})\right): 7.74\left(\mathrm{~d}, \mathrm{~J}=8.4 \mathrm{~Hz}, 2 \mathrm{H}, \mathrm{H}-2^{\prime} \& \mathrm{H}-6^{\prime}\right), 7.33\left(\mathrm{~d}=8.4 \mathrm{~Hz}, 2 \mathrm{H}, \mathrm{H}-3^{\prime}\right.$ \& H-5'), 7.17-7.23 (m, 5H, H-2" to H-6" $), 4.92\left(\mathrm{~s}, 2 \mathrm{H}, \mathrm{H}-7^{\prime \prime}\right), 3.95-3.86\left(\mathrm{~m}, 1 \mathrm{H}, \mathrm{H}_{e}-5\right), 3.77(\mathrm{dd}, \mathrm{J}=6.8,7.6 \mathrm{~Hz}, 1 \mathrm{H}$, $\left.\mathrm{H}_{a}-6\right), 3.68\left(\mathrm{dd}, \mathrm{J}=7.2,8.0 \mathrm{~Hz}, 1 \mathrm{H}, \mathrm{H}_{b}-6\right), 3.14-3.11(\mathrm{~m}, 1 \mathrm{H}, \mathrm{H}-2), 2.87-2.82\left(\mathrm{~m}, 1 \mathrm{H}, \mathrm{H}_{a}-5\right), 2.39\left(\mathrm{~s}, 3 \mathrm{H}, \mathrm{CH}_{3}-7^{\prime}\right)$, 1.94-1.81 (m, 3H, $\mathrm{H}_{e}-3$ \& H-4), 1.62-1.51 (m, 1H, $\left.\mathrm{H}_{a}-3\right)$; EIMS (m/z): 345, 254, 190, 155, 91, 85, 76, 71, 65, 50.

\subsubsection{N-(2-Chlorobenzyl)-N-(tetrahydrofuran-2-ylmethyl)-4-methylbenzenesulfonamide (5y)}

Yellow sticky liquid; Yield: 60\%; M.F.: $\mathrm{C}_{19} \mathrm{H}_{22} \mathrm{ClNO}_{3}$ S; M.W.: $379 \mathrm{gmol}^{-1}$; IR $\left(\mathrm{KBr}, v_{\max }\left(\mathrm{cm}^{-1}\right)\right)$ : 3087, 1614, 1395, 1176, 698; ${ }^{1} \mathrm{H}-\mathrm{NMR}\left(400 \mathrm{MHz}, \mathrm{CDCl}_{3}, \delta(\mathrm{ppm})\right): 7.72\left(\mathrm{~d}, \mathrm{~J}=8.4 \mathrm{~Hz}, 2 \mathrm{H}, \mathrm{H}-2^{\prime}\right.$ \& H-6 $\left.{ }^{\prime}\right), 7.32(\mathrm{dd}, \mathrm{J}=$ 3.2, $\left.8.0 \mathrm{~Hz}, 1 \mathrm{H}, \mathrm{H}-3^{\prime \prime}\right), 7.32\left(\mathrm{~d}, \mathrm{~J}=8.4 \mathrm{~Hz}, 2 \mathrm{H}, \mathrm{H}-3^{\prime} \& \mathrm{H}-5^{\prime}\right), 7.14\left(\mathrm{dd}, \mathrm{J}=3.6,8.8 \mathrm{~Hz}, 1 \mathrm{H}, \mathrm{H}-6^{\prime \prime}\right), 7.13-7.09(\mathrm{~m}, 2 \mathrm{H}$, $\left.\mathrm{H}-4^{\prime \prime} \& \mathrm{H}-5^{\prime \prime}\right), 4.47\left(\mathrm{~s}, 2 \mathrm{H}, \mathrm{H}-7^{\prime \prime}\right), 3.97-3.84\left(\mathrm{~m}, 1 \mathrm{H}, \mathrm{H}_{e}-5\right), 3.75\left(\mathrm{dd}, \mathrm{J}=6.8,8.0 \mathrm{~Hz}, 1 \mathrm{H}, \mathrm{H}_{a}-6\right), 3.70(\mathrm{dd}, \mathrm{J}=6.8$, $\left.8.4 \mathrm{~Hz}, 1 \mathrm{H}, \mathrm{H}_{b}-6\right), 3.12-3.09(\mathrm{~m}, 1 \mathrm{H}, \mathrm{H}-2), 2.85-2.82\left(\mathrm{~m}, 1 \mathrm{H}, \mathrm{H}_{a}-5\right), 2.42\left(\mathrm{~s}, 3 \mathrm{H}, \mathrm{CH}_{3}-7^{\prime}\right), 1.95-1.83\left(\mathrm{~m}, 3 \mathrm{H}, \mathrm{H}_{e}-3\right.$ \& H-4), 1.64-1.51 (m, 1H, $\left.\mathrm{H}_{a}-3\right)$; EIMS (m/z): 381, 379, 254, 190, 155, 125, 99, 91, 90, 85, 76, 71, 65, 50.

\subsubsection{N-(4-Chlorobenzyl)-N-(tetrahydrofuran-2-ylmethyl)-4-methylbenzenesulfonamide (5z)}

. Light green sticky liquid; Yield: 65\%; M.F.: $\mathrm{C}_{19} \mathrm{H}_{22} \mathrm{ClNO}_{3}$ S; M.W.: $379 \mathrm{gmol}^{-1}$; IR $\left(\mathrm{KBr}, v_{\max }\left(\mathrm{cm}^{-1}\right)\right)$ : 3026, 1610, 1357, 1147, 703; ${ }^{1} \mathrm{H}-\mathrm{NMR}\left(400 \mathrm{MHz}, \mathrm{CDCl}_{3}, \delta(\mathrm{ppm})\right): 7.74\left(\mathrm{~d}, \mathrm{~J}=8.4 \mathrm{~Hz}, 2 \mathrm{H}, \mathrm{H}-2^{\prime}\right.$ \& H-6'), 7.52 (d, $\mathrm{J}=8.4 \mathrm{~Hz}, 2 \mathrm{H}, \mathrm{H}-3^{\prime \prime}$ \& H-5") $, 7.26\left(\mathrm{~d}, \mathrm{~J}=8.0 \mathrm{~Hz}, 2 \mathrm{H}, \mathrm{H}-3^{\prime} \& \mathrm{H}-5^{\prime}\right), 7.07\left(\mathrm{~d}, \mathrm{~J}=8.4 \mathrm{~Hz}, 2 \mathrm{H}, \mathrm{H}-2^{\prime \prime}\right.$ \& H-6" $\left.{ }^{\prime \prime}\right), 4.83$ (s, 2H, H-7"), 3.91-3.84 (m, 1H, $\left.\mathrm{H}_{e}-5\right), 3.79\left(\mathrm{dd}, \mathrm{J}=6.8,7.6 \mathrm{~Hz}, 1 \mathrm{H}, \mathrm{H}_{a}-6\right), 3.69\left(\mathrm{dd}, \mathrm{J}=7.2,7.6 \mathrm{~Hz}, 1 \mathrm{H}, \mathrm{H}_{b}-6\right)$, 3.13-3.09 (m, 1H, H-2), 2.87-2.83 (m, $\left.1 \mathrm{H}, \mathrm{H}_{a}-5\right), 2.43\left(\mathrm{~s}, 3 \mathrm{H}, \mathrm{CH}_{3}-7^{\prime}\right), 1.95-1.84\left(\mathrm{~m}, 3 \mathrm{H}, \mathrm{H}_{e}-3\right.$ \& $\left.\mathrm{H}-4\right), 1.64-1.57$ (m, 1H, $\left.\mathrm{H}_{a}-3\right)$; EIMS (m/z): 381, 379, 254, 190, 155, 125, 99, 91, 85, 76, 71, 65, 50. 


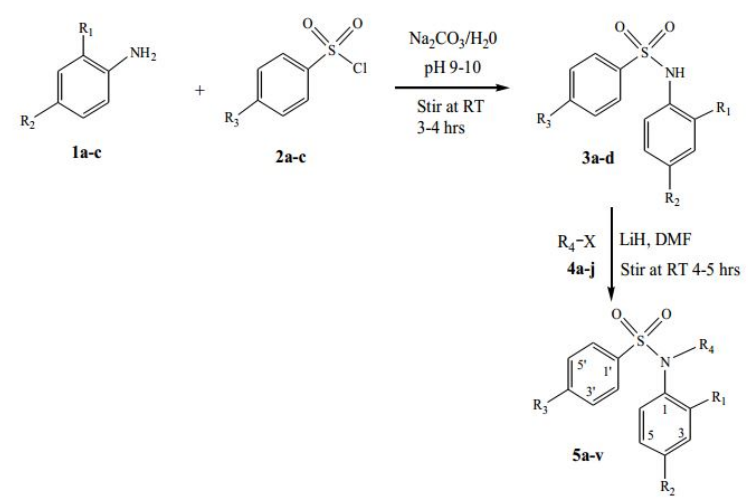

Figure 1. N-Substituted derivatives (5a-v) of N-(2/4-substitutedphenyl)-4- substitutedbenzenesulfonamide (3a-d)

\subsubsection{N-(2-Phenylethyl)-N-(tetrahydrofuran-2-ylmethyl)-4-methylbenzenesulfonamide (5aa)}

Yellowish gummy liquid; Yield: 69\%; M.F.: $\mathrm{C}_{20} \mathrm{H}_{25} \mathrm{NO}_{3}$ S; M.W.: $359 \mathrm{gmol}^{-1}$; IR $\left(\mathrm{KBr}, v_{\max }\left(\mathrm{cm}^{-1}\right)\right): 2975$, 1610, 1426, 1153; ${ }^{1} \mathrm{H}-\mathrm{NMR}\left(400 \mathrm{MHz}, C D C l_{3}, \delta(\mathrm{ppm})\right): 7.76\left(\mathrm{~d}, \mathrm{~J}=8.4 \mathrm{~Hz}, 2 \mathrm{H}, \mathrm{H}-2^{\prime} \& \mathrm{H}-6^{\prime}\right), 7.26(\mathrm{~d}, \mathrm{~J}=8.0 \mathrm{~Hz}$, $\left.2 \mathrm{H}, \mathrm{H}-3^{\prime} \& \mathrm{H}-5^{\prime}\right), 7.24\left(\mathrm{t}, \mathrm{J}=7.6 \mathrm{~Hz}, 2 \mathrm{H}, \mathrm{H}-3^{\prime \prime} \& \mathrm{H}-5^{\prime \prime}\right), 7.17\left(\mathrm{t}, \mathrm{J}=7.2 \mathrm{~Hz}, 1 \mathrm{H}, \mathrm{H}-4^{\prime \prime}\right), 7.09(\mathrm{~d}, \mathrm{~J}=7.6 \mathrm{~Hz}, 2 \mathrm{H}$, $\left.\mathrm{H}-2^{\prime \prime} \& \mathrm{H}-6^{\prime \prime}\right), 3.90-3.83\left(\mathrm{~m}, 1 \mathrm{H}, \mathrm{H}_{e}-5\right), 3.75\left(\mathrm{dd}, \mathrm{J}=6.8,8.4 \mathrm{~Hz}, 1 \mathrm{H}, \mathrm{H}_{a}-6\right), 3.73\left(\mathrm{t}, \mathrm{J}=7.6 \mathrm{~Hz}, 2 \mathrm{H}, \mathrm{H}-8^{\prime \prime}\right), 3.67$ $\left(\mathrm{dd}, \mathrm{J}=6.8,8.0 \mathrm{~Hz} 1 \mathrm{H}, \mathrm{H}_{b}-6\right), 3.13-3.08(\mathrm{~m}, 1 \mathrm{H}, \mathrm{H}-2), 2.87-2.85\left(\mathrm{~m}, 1 \mathrm{H}, \mathrm{H}_{a}-5\right), 2.67\left(\mathrm{t}, \mathrm{J}=7.2 \mathrm{~Hz}, 2 \mathrm{H}, \mathrm{H}-7^{\prime \prime}\right), 2.39$ (s, 3H, $\left.\mathrm{CH}_{3}-7^{\prime}\right), 1.92-1.83$ (m, 3H, $\mathrm{H}_{e}-3$ \& H-4), 1.68-1.57 (m, 1H, $\left.\mathrm{H}_{a}-3\right)$; EIMS (m/z): 359, 254, 190, 155, 105, $99,91,85,76,71,65,57,51,50$.

\subsubsection{N-(3-Phenylpropyl)-N-(tetrahydrofuran-2-ylmethyl)-4-methylbenzenesulfonamide (5bb)}

Golden gummy material; Yield: 71\%; M.F.: $\mathrm{C}_{21} \mathrm{H}_{27} \mathrm{NO}_{3}$ S; M.W.: $373 \mathrm{gmol}^{-1}$; IR (KBr, $\left.v_{\max }\left(\mathrm{cm}^{-1}\right)\right)$ : 2987, 1607, 1407, 1171; ${ }^{1} \mathrm{H}-\mathrm{NMR}(400 \mathrm{MHz}, \mathrm{CDCl} 3, \delta(\mathrm{ppm})): 7.68\left(\mathrm{~d}, \mathrm{~J}=8.4 \mathrm{~Hz}, 2 \mathrm{H}, \mathrm{H}-2^{\prime} \& \mathrm{H}-6^{\prime}\right), 7.26(\mathrm{~d}, \mathrm{~J}=8.0 \mathrm{~Hz}$, $\left.2 \mathrm{H}, \mathrm{H}-3^{\prime} \& \mathrm{H}-5^{\prime}\right), 7.21\left(\mathrm{t}, \mathrm{J}=8.0 \mathrm{~Hz}, 2 \mathrm{H}, \mathrm{H}-3^{\prime \prime} \& \mathrm{H}-5^{\prime \prime}\right), 7.19\left(\mathrm{t}, \mathrm{J}=7.6 \mathrm{~Hz}, 1 \mathrm{H}, \mathrm{H}-4^{\prime \prime}\right), 7.03(\mathrm{~d}, \mathrm{~J}=7.6 \mathrm{~Hz}, 2 \mathrm{H}$, $\mathrm{H}-2^{\prime \prime}$ \& H-6"), 3.95-3.89 (m, 1H, $\left.\mathrm{H}_{e}-5\right), 3.77\left(\mathrm{dd}, \mathrm{J}=6.8,8.0 \mathrm{~Hz}, 1 \mathrm{H}, \mathrm{H}_{a}-6\right), 3.75\left(\mathrm{t}, \mathrm{J}=7.2 \mathrm{~Hz}, 2 \mathrm{H}, \mathrm{H}-9^{\prime \prime}\right), 3.70$ $\left(\mathrm{dd}, \mathrm{J}=7.2,8.0 \mathrm{~Hz}, 1 \mathrm{H}, \mathrm{H}_{b}-6\right), 3.12-3.10(\mathrm{~m}, 1 \mathrm{H}, \mathrm{H}-2), 2.84-2.82\left(\mathrm{~m}, 1 \mathrm{H}, \mathrm{H}_{a}-5\right), 2.67\left(\mathrm{t}, \mathrm{J}=7.6 \mathrm{~Hz}, 2 \mathrm{H}, \mathrm{H}-7^{\prime \prime}\right), 2.44$ (s, 3H, $\left.\mathrm{CH}_{3}-7^{\prime}\right), 2.02\left(\right.$ qui, J = 7.2 Hz, $\left.2 \mathrm{H}, \mathrm{CH}_{2}-8^{\prime \prime}\right), 1.91-1.82\left(\mathrm{~m}, 3 \mathrm{H}, \mathrm{H}_{e}-3\right.$ \& $\left.\mathrm{H}-4\right), 1.64-1.55\left(\mathrm{~m}, 1 \mathrm{H}, \mathrm{H}_{a}-3\right)$; EIMS (m/z): 373, 254, 190, 155, 119, 105, 91, 99, 85, 76, 71, 65, 57, 51.

\subsection{Antibacterial activity assay}

The antibacterial activity was performed in sterile 96-wells microplates under aseptic environments. The method is based on the principle that microbial cell number increases as the microbial growth proceeds in a log phase of growth which results in increased absorbance of broth medium [12].

\subsection{Statistical analysis}

All the measurements were accounted in triplicate and statistical analysis was performed by Microsoft Excel 2010. Results are presented as mean \pm SEM. EZ-Fit kinetics software (Perrella Scientific Inc. Amherst, USA) was used to find Minimum Inhibitory Concentration (MIC) values.

\section{Results and Discussion}

N-Substituted derivatives of sulfonamides were synthesized by protocol elaborated in Scheme given in Figure 1 and Scheme given in Figure 2. The list of different substituents is given in Table 1. In experimental section, the procedures of reaction along with reaction conditions are discussed. The proposed structures of all the synthesized compounds were analyzed through spectral analysis using ${ }^{1} \mathrm{H}-\mathrm{NMR}$, IR and EIMS techniques. These compounds were further screened against gram-bacteria with an objective to introduce new potent compounds against these bacteria in the field of pharmacology. 


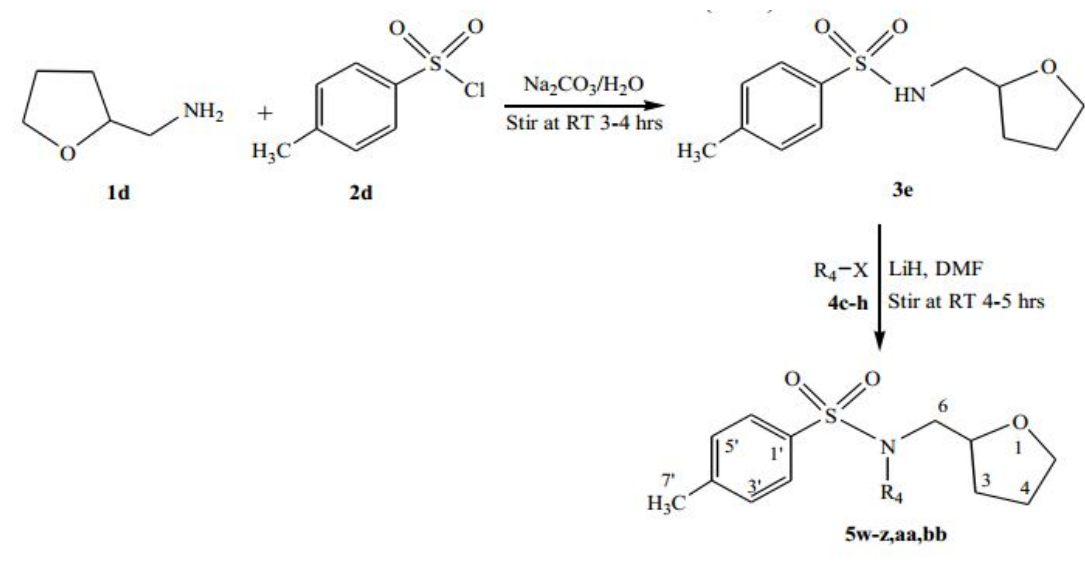

Figure 2. N-Substituted derivatives $(5 \mathrm{w}-\mathrm{z}, \mathrm{aa}, \mathrm{bb})$ of N- (tetrahydrofuran- 2- ylmethyl)-4methylbenzenesulfonamide (3e)

Table 1. Different varying groups of synthesized derivatives

\begin{tabular}{|c|c|c|c|c|}
\hline Compound & R1 & $\mathbf{R} 2$ & R3 & $\mathbf{R 4}$ \\
\hline $5 a$ & $-\mathrm{C}_{2} \mathrm{H}_{5}$ & $-\mathrm{H}$ & $-\mathrm{NHCOCH}_{3}$ & $-\mathrm{CH}\left(\mathrm{CH}_{3}\right)_{2}$ \\
\hline $5 b$ & $-\mathrm{C}_{2} \mathrm{H}_{5}$ & $-\mathrm{H}$ & $-\mathrm{NHCOCH}_{3}$ & $-\mathrm{CH}\left(\mathrm{CH}_{3}\right) \mathrm{CH}_{2} \mathrm{CH}_{3}$ \\
\hline $5 c$ & $-\mathrm{C}_{2} \mathrm{H}_{5}$ & $-\mathrm{H}$ & $-\mathrm{NHCOCH}_{3}$ & $-\mathrm{CH}_{2}\left(\mathrm{CH}_{2}\right)_{3} \mathrm{CH}_{3}$ \\
\hline $5 d$ & $-\mathrm{C}_{2} \mathrm{H}_{5}$ & $-\mathrm{H}$ & $-\mathrm{NHCOCH}_{3}$ & $-\mathrm{CH}_{2} \mathrm{C}_{6} \mathrm{H}_{5}$ \\
\hline $5 e$ & $-\mathrm{C}_{2} \mathrm{H}_{5}$ & $-\mathrm{H}$ & $-\mathrm{NHCOCH}_{3}$ & $-\mathrm{CH}_{2}-2-\mathrm{ClC}_{6} \mathrm{H}_{5}$ \\
\hline $5 f$ & $-\mathrm{C}_{2} \mathrm{H}_{5}$ & $-\mathrm{H}$ & $-\mathrm{NHCOCH}_{3}$ & $-\mathrm{CH}_{2}-4-\mathrm{ClC}_{6} \mathrm{H}_{5}$ \\
\hline $5 g$ & $-\mathrm{H}$ & $-\mathrm{C}_{2} \mathrm{H}_{5}$ & $-\mathrm{Cl}$ & $-\mathrm{CH}_{2} \mathrm{CH}_{2} \mathrm{Cl}$ \\
\hline $5 \mathrm{~h}$ & $-\mathrm{H}$ & $-\mathrm{C}_{2} \mathrm{H}_{5}$ & $-\mathrm{Cl}$ & $-\mathrm{CH}_{2} \mathrm{CH}_{2} \mathrm{Br}$ \\
\hline $5 \mathbf{i}$ & $-\mathrm{H}$ & $-\mathrm{C}_{2} \mathrm{H}_{5}$ & $-\mathrm{Cl}$ & $-\mathrm{CH}_{2}-2-\mathrm{ClC}_{6} \mathrm{H}_{5}$ \\
\hline $5 \mathbf{j}$ & $-\mathrm{H}$ & $-\mathrm{C}_{2} \mathrm{H}_{5}$ & $-\mathrm{Cl}$ & $-\mathrm{CH}_{2} \mathrm{CH}_{2} \mathrm{C}_{6} \mathrm{H}_{5}$ \\
\hline $5 \mathrm{k}$ & $-\mathrm{H}$ & $-\mathrm{C}_{2} \mathrm{H}_{5}$ & $-\mathrm{Cl}$ & $-\mathrm{CH}_{2} \mathrm{CH}_{2} \mathrm{CH}_{2} \mathrm{C}_{6} \mathrm{H}_{5}$ \\
\hline 51 & $-\mathrm{H}$ & $-\mathrm{OC}_{2} \mathrm{H}_{5}$ & $-\mathrm{Cl}$ & $-\mathrm{CH}_{2} \mathrm{CH}_{2} \mathrm{Cl}$ \\
\hline $5 \mathrm{~m}$ & $-\mathrm{H}$ & $-\mathrm{OC}_{2} \mathrm{H}_{5}$ & $-\mathrm{Cl}$ & $-\mathrm{CH}_{2} \mathrm{CH}_{2} \mathrm{Br}$ \\
\hline $5 n$ & $-\mathrm{H}$ & $-\mathrm{OC}_{2} \mathrm{H}_{5}$ & $-\mathrm{Cl}$ & $-\mathrm{CH}_{2}-2-\mathrm{ClC}_{6} \mathrm{H}_{5}$ \\
\hline 50 & $-\mathrm{H}$ & $-\mathrm{OC}_{2} \mathrm{H}_{5}$ & $-\mathrm{Cl}$ & $-\mathrm{CH}_{2} \mathrm{CH}_{2} \mathrm{C}_{6} \mathrm{H}_{5}$ \\
\hline $5 p$ & $-\mathrm{H}$ & $-\mathrm{OC}_{2} \mathrm{H}_{5}$ & $-\mathrm{Cl}$ & $-\mathrm{CH}_{2} \mathrm{CH}_{2} \mathrm{CH}_{2} \mathrm{C}_{6} \mathrm{H}_{5}$ \\
\hline $5 q$ & $-\mathrm{H}$ & $-\mathrm{OC}_{2} \mathrm{H}_{5}$ & $-\mathrm{H}$ & $-\mathrm{CH}\left(\mathrm{CH}_{3}\right)_{2}$ \\
\hline $5 r$ & $-\mathrm{H}$ & $-\mathrm{OC}_{2} \mathrm{H}_{5}$ & $-\mathrm{H}$ & $-\mathrm{CH}\left(\mathrm{CH}_{3}\right) \mathrm{CH}_{2} \mathrm{CH}_{3}$ \\
\hline $5 s$ & $-\mathrm{H}$ & $-\mathrm{OC}_{2} \mathrm{H}_{5}$ & $-\mathrm{H}$ & $-\mathrm{CH}_{2}\left(\mathrm{CH}_{2}\right)_{3} \mathrm{CH}_{3}$ \\
\hline $5 t$ & $-\mathrm{H}$ & $-\mathrm{OC}_{2} \mathrm{H}_{5}$ & $-\mathrm{H}$ & $-\mathrm{CH}_{2} \mathrm{C}_{6} \mathrm{H}_{5}$ \\
\hline $5 \mathbf{u}$ & $-\mathrm{H}$ & $-\mathrm{OC}_{2} \mathrm{H}_{5}$ & $-\mathrm{H}$ & $-\mathrm{CH}_{2}-2-\mathrm{ClC}_{6} \mathrm{H}_{5}$ \\
\hline $5 \mathbf{v}$ & $-\mathrm{H}$ & $-\mathrm{OC}_{2} \mathrm{H}_{5}$ & $-\mathrm{H}$ & $-\mathrm{CH}_{2}-4-\mathrm{ClC}_{6} \mathrm{H}_{5}$ \\
\hline $5 w$ & - & - & - & $-\mathrm{CH}_{2}\left(\mathrm{CH}_{2}\right)_{3} \mathrm{CH}_{3}$ \\
\hline $5 x$ & - & - & - & $-\mathrm{CH}_{2} \mathrm{C}_{6} \mathrm{H}_{5}$ \\
\hline $5 y$ & - & - & - & $-\mathrm{CH}_{2}-2-\mathrm{ClC}_{6} \mathrm{H}_{5}$ \\
\hline $5 z$ & - & - & - & $-\mathrm{CH}_{2}-4-\mathrm{ClC}_{6} \mathrm{H}_{5}$ \\
\hline 5 aa & - & - & - & $-\mathrm{CH}_{2} \mathrm{CH}_{2} \mathrm{C}_{6} \mathrm{H}_{5}$ \\
\hline $5 \mathrm{bb}$ & - & - & - & $-\mathrm{CH}_{2} \mathrm{CH}_{2} \mathrm{CH}_{2} \mathrm{C}_{6} \mathrm{H}_{5}$ \\
\hline
\end{tabular}

\subsection{Chemistry}

The 4-acetamido-N-(2-ethylphenyl)benzenesulfonamide (3a) was obtained as Grayish white amorphous solid in a better yield. Affirmation of molecular formula was carried out by EI-MS showing a $[\mathrm{M}]^{+}$ion peak at $\mathrm{m} / \mathrm{z} 318$ stepping to the molecular formula, $\mathrm{C}_{16} \mathrm{H}_{18} \mathrm{~N}_{2} \mathrm{O}_{3} \mathrm{~S}$. Confirmation of molecular formula was carried out by counting the number of protons using integration curves in its ${ }^{1} \mathrm{H}-\mathrm{NMR}$ spectrum. In ${ }^{1} \mathrm{H}-\mathrm{NMR}$ spectrum, the two doublets at $\delta 7.66\left(\mathrm{~d}, \mathrm{~J}=8.8 \mathrm{~Hz}, 2 \mathrm{H}, \mathrm{H}-3^{\prime}, \mathrm{H}-5^{\prime}\right), 7.56\left(\mathrm{~d}, \mathrm{~J}=8.8 \mathrm{~Hz}, 2 \mathrm{H}, \mathrm{H}-2^{\prime}, \mathrm{H}-6^{\prime}\right)$ and one singlet at $\delta$ $2.18\left(\mathrm{~s}, 3 \mathrm{H}, \mathrm{CH}_{3}-8^{\prime}\right)$ were assigned to the eight protons of 4-acetamidobenzenesulfonyl group present in the 
compound. The signals resonating at 7.11-7.10 (m, 4H, H-3 to H-6), $2.31\left(\mathrm{q}, \mathrm{J}=7.6 \mathrm{~Hz}, 2 \mathrm{H}, \mathrm{CH}_{2}-7\right), 2.18(\mathrm{~s}, 3 \mathrm{H}$, $\left.\mathrm{CH}_{3}-8^{\prime}\right)$ and $1.04\left(\mathrm{t}, \mathrm{J}=7.6 \mathrm{~Hz}, 3 \mathrm{H}, \mathrm{CH}_{3}-8\right)$ were allocated to the protons of 2-ethylaniline. Affirmation of the main functional groups present in the molecule was supported by IR spectrum by absorption bands at 3431 $(\mathrm{N} \mathrm{H}), 3016$ (Ar C H), 1666 (Amide $\mathrm{C}=\mathrm{O}), 1586(\mathrm{Ar} \mathrm{C}=\mathrm{C}$ ) and $1411(\mathrm{~S}=\mathrm{O})$. All these evidences collaborated the proposed structure of $\mathbf{3 a}$. The structures of other synthesized compounds $(\mathbf{3} \mathbf{b}-\mathbf{e}, \mathbf{5} \mathbf{a}-\mathbf{z}, \mathbf{a a}, \mathbf{b b})$ were elucidated by using the spectral data, as described in experimental section.

\subsection{Antibacterial activity}

The antibacterial activity was evaluated against gram-bacteria and their results are presented as MIC values in Table 2. S. typhi (-) was moderately inhibited by all the compounds excluding $\mathbf{5 o}$ but $\mathbf{3 b} \mathbf{b} \mathbf{- e}, \mathbf{5 g}, \mathbf{5 h}, \mathbf{5 w}$, $5 \mathbf{y}, 5 \mathbf{z}$ and $5 \mathbf{b b}$ were the most active ones. The starting molecules remained more active in this case in general. The series of compounds bearing heterocyclic tetrahydrofuran ring were the most active ones against this strain. Almost the similar pattern of inhibition was observed for E. coli (-). The most active compounds against this strain were $\mathbf{3 b}-\mathbf{d}, \mathbf{5 i}, \mathbf{5 k}, \mathbf{5 m}, \mathbf{5 n}, \mathbf{5 x}, \mathbf{5 y}$ and $\mathbf{5 b b}$. P. aeruginosa (-) was less efficiently inhibited by these molecules and many of them remained inactive at all. The only considerably highly active three compounds were $\mathbf{3 c}, \mathbf{5 g}$ and 51 . Only ten compounds remained considerably highly efficient against $B$. subtilis $(+)$ and the most of compounds remained moderately highly active. The most active molecules against this strain were

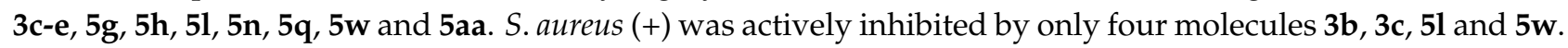
The compounds $\mathbf{5 b}, \mathbf{5 c}, \mathbf{5} \mathbf{u}$ and $\mathbf{5 v}$ remained active only against $S$. typhi (-). Overall the synthesized molecules executed moderately good inhibition potential against the gram-negative strain, S. typhi (-).

\section{Conclusion}

All the molecules were synthesized in excellent yields by a simple benign method as discussed in experimental section in detail. The structures of all molecules were well supported by their spectral data using techniques such as IR, ${ }^{1} \mathrm{H}-\mathrm{NMR}$ and EIMS. The antibacterial activity results of all the synthesized compounds rendered them moderate inhibitors of gram-negative and gram-positive bacteria. Some of compounds were found to be active against all the bacterial strains and some of them were highly active against a certain strain. The list of molecules can be further considered for drug discovery pathway to cure the diseases caused by this bacterial strain.

Author Contributions: All authors contributed equally to the writing of this paper. All authors read and approved the final manuscript.

Conflicts of Interest: “The authors declare no conflict of interest."

\section{References}

[1] Camargo-Ordoñez, A., Moreno-Reyes, C., Olazarán-Santibáñez, F., Martínez-Hernández, S., Bocanegra-García, V., \& Rivera, G. (2011). Efficient synthesis of sulfonamide derivatives on solid supports catalyzed using solvent-free and microwave-assisted methods. Química Nova, 34(5), 787-791.

[2] Azab, M., Youssef, M., \& El-Bordany, E. (2013). Synthesis and antibacterial evaluation of novel heterocyclic compounds containing a sulfonamido moiety. Molecules, 18(1), 832-844.

[3] Boyd, A. E. (1988). Sulfonylurea receptors, ion channels, and fruit flies. Diabetes, 37(7), 847-850.

[4] Supuran, C. T., \& Scozzafava, A. (2000). Carbonic anhydrase inhibitors and their therapeutic potential. Expert Opinion on Therapeutic Patents, 10(5), 575-600.

[5] Kadi, A. A., El-Brollosy, N. R., Al-Deeb, O. A., Habib, E. E., Ibrahim, T. M., \& El-Emam, A. A. (2007). Synthesis, antimicrobial, and anti-inflammatory activities of novel 2-(1-adamantyl)-5-substituted-1, 3, 4-oxadiazoles and 2-(1-adamantylamino)-5-substituted-1, 3, 4-thiadiazoles. European journal of medicinal chemistry, 42(2), $235-242$.

[6] Liou, J. P., Hsu, K. S., Kuo, C. C., Chang, C. Y., \& Chang, J. Y. (2007). A novel oral indoline-sulfonamide agent, $\mathrm{N}$-[1-(4-methoxybenzenesulfonyl)-2, 3-dihydro-1H-indol-7-yl]-isonicotinamide (J30), exhibits potent activity against human cancer cells in vitro and in vivo through the disruption of microtubule. Journal of Pharmacology and Experimental Therapeutics, 323(1), 398-405.

[7] Ghorab, M. M., Ragab, F. A., Heiba, H. I., \& El-Hazek, R. M. (2011). Anticancer and radio-sensitizing evaluation of some new thiazolopyrane and thiazolopyranopyrimidine derivatives bearing a sulfonamide moiety. European journal of medicinal chemistry, 46(10), 5120-5126. 
Table 2. MIC for antibacterial activity of synthesized compounds

\begin{tabular}{|c|c|c|c|c|c|}
\hline & S. typhi (-) & E. coli (-) & P. aeruginosa (-) & B. subtilis (+) & S. aureus (+) \\
\hline $3 a$ & $10.41 \pm 0.37$ & $12.42 \pm 0.75$ & $14.73 \pm 0.91$ & $11.78 \pm 0.94$ & $12.73 \pm 0.81$ \\
\hline $3 b$ & $9.03 \pm 0.47$ & $7.98 \pm 0.35$ & $11.25 \pm 0.77$ & $15.91 \pm 0.22$ & $9.09 \pm 0.48$ \\
\hline $3 c$ & $9.21 \pm 0.01$ & $8.11 \pm 0.62$ & $9.24 \pm 0.22$ & $8.12 \pm 0.28$ & $9.68 \pm 0.33$ \\
\hline $3 d$ & $8.21 \pm 0.73$ & $9.72 \pm 1.05$ & $14.41 \pm 0.52$ & $9.37 \pm 1.00$ & $14.31 \pm 0.18$ \\
\hline $3 e$ & $9.16 \pm 0.27$ & $10.32 \pm 0.45$ & $12.39 \pm 0.82$ & $9.07 \pm 1.02$ & $12.63 \pm 0.48$ \\
\hline $5 a$ & $11.27 \pm 0.33$ & $11.43 \pm 0.17$ & $16.42 \pm 0.55$ & $13.38 \pm 0.57$ & $12.56 \pm 0.25$ \\
\hline $5 b$ & $17.11 \pm 0.31$ & - & - & - & - \\
\hline $5 c$ & $16.82 \pm 0.19$ & - & - & - & - \\
\hline $5 d$ & $17.23 \pm 0.62$ & $12.41 \pm 0.16$ & - & $16.24 \pm 0.24$ & - \\
\hline $5 e$ & $16.24 \pm 0.23$ & - & - & - & - \\
\hline $5 f$ & $12.81 \pm 0.45$ & $18.01 \pm 0.15$ & $11.37 \pm 0.86$ & $13.62 \pm 0.12$ & - \\
\hline $5 g$ & $7.85 \pm 0.33$ & $14.68 \pm 0.37$ & $9.72 \pm 0.63$ & $7.13 \pm 0.38$ & $16.91 \pm 0.43$ \\
\hline $5 h$ & $7.61 \pm 0.12$ & - & $10.94 \pm 0.12$ & $8.00 \pm 0.34$ & - \\
\hline $5 i$ & $14.81 \pm 0.35$ & $7.98 \pm 0.65$ & - & - & $10.59 \pm 0.54$ \\
\hline $5 \mathbf{j}$ & $15.39 \pm 0.55$ & $16.24 \pm 0.16$ & - & $14.95 \pm 0.90$ & - \\
\hline $5 k$ & $12.63 \pm 1.09$ & $8.76 \pm 0.41$ & - & $14.75 \pm 0.84$ & $11.48 \pm 0.88$ \\
\hline 51 & $12.59 \pm 0.36$ & $11.49 \pm 0.46$ & $9.89 \pm 0.01$ & $9.01 \pm 0.78$ & $9.23 \pm 0.59$ \\
\hline $5 \mathrm{~m}$ & $14.76 \pm 0.91$ & $9.58 \pm 0.24$ & - & $12.31 \pm 0.43$ & $15.97 \pm 0.64$ \\
\hline $5 n$ & $9.54 \pm 0.24$ & $8.97 \pm 0.05$ & $13.53 \pm 0.73$ & $9.79 \pm 0.37$ & $13.10 \pm 0.33$ \\
\hline 50 & - & $14.60 \pm 0.15$ & - & $17.26 \pm 1.01$ & - \\
\hline $5 p$ & $10.12 \pm 0.41$ & $13.29 \pm 0.48$ & $15.42 \pm 0.11$ & $10.00 \pm 0.51$ & $12.00 \pm 0.61$ \\
\hline $5 q$ & $10.16 \pm 0.53$ & $10.21 \pm 0.05$ & $13.52 \pm 0.25$ & $8.50 \pm 0.53$ & $13.64 \pm 0.93$ \\
\hline $5 r$ & $10.09 \pm 0.18$ & $10.91 \pm 0.48$ & $14.81 \pm 0.92$ & - & $13.09 \pm 0.74$ \\
\hline $5 s$ & $10.35 \pm 0.79$ & $10.48 \pm 0.41$ & $14.33 \pm 0.91$ & $11.75 \pm 0.74$ & $12.09 \pm 0.81$ \\
\hline $5 t$ & $10.60 \pm 0.43$ & $12.61 \pm 1.00$ & $15.53 \pm 0.31$ & $14.16 \pm 0.53$ & $15.52 \pm 0.65$ \\
\hline $5 \mathbf{u}$ & $15.89 \pm 0.86$ & - & - & - & - \\
\hline $5 v$ & $17.39 \pm 0.92$ & - & - & - & - \\
\hline $5 w$ & $9.82 \pm 0.19$ & $10.01 \pm 0.36$ & $13.39 \pm 0.15$ & $9.47 \pm 0.90$ & $9.01 \pm 0.76$ \\
\hline $5 x$ & $10.11 \pm 0.68$ & $9.28 \pm 0.15$ & $14.98 \pm 0.95$ & $11.25 \pm 0.82$ & $12.43 \pm 1.05$ \\
\hline $5 y$ & $9.75 \pm 0.12$ & $9.88 \pm 0.39$ & $15.08 \pm 0.49$ & $10.45 \pm 0.84$ & $11.22 \pm 0.60$ \\
\hline $5 z$ & $9.44 \pm 0.69$ & $11.45 \pm 0.77$ & $15.19 \pm 0.92$ & $10.27 \pm 0.90$ & $10.82 \pm 0.81$ \\
\hline $5 \mathbf{a a}$ & $10.11 \pm 0.18$ & $10.32 \pm 0.53$ & $12.44 \pm 1.41$ & $9.21 \pm 0.71$ & $10.38 \pm 0.14$ \\
\hline $5 b b$ & $9.80 \pm 0.61$ & $9.25 \pm 0.34$ & $17.19 \pm 0.35$ & $10.62 \pm 0.68$ & $12.94 \pm 0.97$ \\
\hline Ciprofloxacin & $7.23 \pm 0.71$ & $8.21 \pm 0.11$ & $7.58 \pm 0.19$ & $7.52 \pm 0.67$ & $7.03 \pm 0.53$ \\
\hline
\end{tabular}

[8] Zoumpoulakis, P., Camoutsis, C., Pairas, G., Sokovic, M., Glamoclija, J., Potamitis, C., \& Pitsas, A. (2012). Synthesis of novel sulfonamide-1, 2, 4-triazoles, 1, 3, 4-thiadiazoles and 1,3,4-oxadiazoles, as potential antibacterial and antifungal agents. Biological evaluation and conformational analysis studies. Bioorganic E medicinal chemistry, 20(4), 1569-1583.

[9] Chen, Z., Xu, W., Liu, K., Yang, S., Fan, H., Bhadury, P. S., ... \& Zhang, Y. (2010). Synthesis and Antiviral Activity of 5-(4-Chlorophenyl)-1, 3, 4-Thiadiazole Sulfonamides. Molecules, 15(12), 9046-9056.

[10] Hruska, K., \& Franek, M. (2012). Sulfonamides in the environment: a review and a case report. Vet Med, 57(1), 1-35.

[11] Abbas, N., Abbasi, M. A., Khalid, H., Khan, K. M., Ashraf, M., Ahmad, I., \& Ejaz, S. A. (2013). Synthesis, Spectral Characterization and Biological Screening of N-Substituted Derivatives of N-(1-Hydroxy-2-methylpropan-2-yl) benzenesulfonamide. Asian Journal of Chemistry, 25(6), 3289

[12] Kaspady, M., Narayanaswamy, V. K., Raju, M., \& Rao, G. K. (2009). Synthesis, antibacterial activity of 2, 4-disubstituted oxazoles and thiazoles as bioisosteres. Letters in Drug Design E Discovery, 6(1), 21-28. 